\title{
Nanoindentation and incipient plasticity
}

\author{
E. B. Tadmor, ${ }^{\text {a) }}$ R. Miller, ${ }^{\text {b) }}$ and R. Phillips ${ }^{\text {c) }}$ \\ Division of Engineering, Brown University, Providence, Rhode Island 02912 \\ M. Ortiz \\ Department of Aeronautics, California Institute of Technology, Pasadena, California 91125
}

(Received 19 August 1998; accepted 1 March 1999)

This paper presents a large-scale atomic resolution simulation of nanoindentation into a thin aluminum film using the recently introduced quasicontinuum method. The purpose of the simulation is to study the initial stages of plastic deformation under the action of an indenter. Two different crystallographic orientations of the film and two different indenter geometries (a rectangular prism and a cylinder) are studied. We obtain both macroscopic load versus indentation depth curves, as well as microscopic quantities, such as the Peierls stress and density of geometrically necessary dislocations beneath the indenter. In addition, we obtain detailed information regarding the atomistic mechanisms responsible for the macroscopic curves. A strong dependence on geometry and orientation is observed. Two different microscopic mechanisms are observed to accommodate the applied loading: (i) nucleation and subsequent propagation into the bulk of edge dislocation dipoles and (ii) deformation twinning.

\section{INTRODUCTION}

As mechanical systems continue to decrease in size and begin to approach atomic length scales, it is becoming important to develop experimental and corresponding theoretical tools to characterize material properties at these scales. One such experimental technique which has become popular due to its relative simplicity is nanoindentation. In this procedure an indenter with dimensions of the order of tens of nanometers is pressed into the surface of a solid. Nanoindentation has now become a standard technique for evaluating the mechanical properties of thin films. ${ }^{1}$ It can also be a useful tool for studying the onset of plastic flow in small volumes, a phenomenon which can play a significant role in macroscopic deformation processes such as adhesion, friction, and fracture. ${ }^{2}$

The nanoindentation test is basically an extension of traditional hardness and microhardness tests to very small scales. The classical tests offer a reasonably unambiguous measure of the hardness or mean pressure beneath the indenter for a given load which can then be related to the yield strength of the material through semiempirical relations. ${ }^{3,4}$ The assumption here is that a large plastic region forms beneath the indenter which can be treated approximately through plastic slip line theories

\footnotetext{
a)Present address: Faculty of Mechanical Engineering, TechnionIsrael Institute of Technology, 32000 Haifa, Israel.

b)Present address: Department of Mechanical Engineering, University of Saskatchewan, Saskatoon, Saskatchewan, S7N 5A9, Canada.

${ }^{c}$ Address all correspondence to this author.

e-mail: phillips@engin.brown.edu
}

or more exactly by computation. Empirical corrections are sometimes necessary to account for effects such as strain hardening and deviations of the indenter from its nominal geometry.

In nanoindentation this relative clarity is lost. At the very small scales and loads common to these experiments the deformation is characterized by discrete dislocation nucleation events and the subsequent interaction of the small numbers of dislocations that have been generated. ${ }^{5}$ This is not the large-scale plasticity observed at the macroscopic scale. It is also not clear what role other mechanisms such as diffusion and block slip ${ }^{2}$ play in this small-scale incipient plasticity. Interpretation is further complicated by the fact that the response can be highly dependent on the indenter geometry and its orientation relative to the specimen crystallography. It can also be strongly influenced by additional factors such as surface effects, ${ }^{2,6}$ substrate effects, ${ }^{7}$ grain effects, ${ }^{8}$ and pre-existing defect populations. ${ }^{9}$

Interpretation of nanoindentation tests may be facilitated by a clearer understanding of the processes taking place during the test. In recent years there have been a number of molecular dynamics (MD) simulations of nanoindentation ${ }^{2,10-12}$ which have led to greater insight into the microscopics of nanoindentation. Due to the computational intensity of the problem many of these simulations were limited to very small model sizes (cubes of only tens of atoms on a side) or very high loading rates, or both. In this work we make use of the recently developed quasicontinuum method ${ }^{13-17}$ which allows for the modeling of systems with dimensions of the order of microns and thus minimizes the possibility 
of the contamination of the results by the boundary conditions arising from the small model size. The issue of loading rate is sidestepped since the simulation is carried out in quasistatic fashion, by determining a series of static equilibrium states, each corresponding to a different load. As long as the load increment is kept sufficiently small, the results are independent of the loading steps.

In this paper we focus on incipient plasticity, the very initial stages of plastic activity, in an aluminum thin film subjected to nanoindentation. We investigate the mechanisms whereby dislocations are nucleated, their subsequent interactions, and the effects of indenter geometry and film crystallography. We find that depending on the crystallography and geometry, completely different microscopic mechanisms are observed with correspondingly different macroscopic manifestations. For a description of the technical details of applying quasicontinuum to study nanoindentation problems, see Ref. 17.

\section{METHODOLOGY}

The quasicontinuum methodology used here is a mixed continuum and atomistic method developed to study problems in the mechanics of materials where multiple scales operate simultaneously. It was originally introduced $^{14,15}$ to study single crystal mechanics and later extended ${ }^{16,17}$ to treat polycrystals and polyphase materials. The basic idea is that in a crystal undergoing mechanical deformation the majority of the lattice experiences a slowly varying deformation on the atomic scale which is well characterized by the continuum approximation. It is only in the vicinity of defects or in the presence of mechanical manipulations on the order of the lattice spacing where discrete atomic effects generally become important. There is thus no need to explicitly treat every single atom in the crystal as is done in standard lattice statics and molecular dynamics approaches.

Within the quasicontinuum method the solution is to select a small subset of the total collection of atoms to represent the energetics of the whole. The crystal is then divided into disjoint cells each containing a single one of these selected atoms whose energetics are assumed to represent those of all other atoms in its cell. Thus if the exact energy of a collection of $N$ atoms is given by,

$$
E_{\text {exact }}=\sum_{i=1}^{N} E_{i},
$$

where $E_{i}$ is the energy of atom $i$, then within the quasicontinuum method a reduced energy potential is defined, such that,

$$
E_{\text {reduced }}=\sum_{i=1}^{R} n_{i} E_{i}
$$

where $R \ll N$ is the number of representative atoms in the selected subset and $n_{i}$ is the number of atoms represented by atom $i$. Clearly, when all atoms are selected to be representative atoms, the exact description is recovered.

This reduced atomic description is stored on a finite element mesh $^{18}$ whose nodes coincide with the representative atom positions. The degrees of freedom of the system are the displacements of the representative atom nodes. The positions of all other atoms in the crystal, which are not explicitly accounted for, can be obtained by finite element interpolation. This becomes necessary when computing the energies of the representative atoms which depend on the positions of their neighboring atoms.

To compute the energies of the representative atoms the embedded atom method (EAM) ${ }^{19}$ is employed. In this scheme the energy of an atom is computed from the relative positions of all other atoms that fall within some specified cutoff, using the relation,

$$
E_{i}=\frac{1}{2} \sum_{j} \phi\left(r_{i j}\right)+U\left(\rho_{i}\right),
$$

where $r_{i j}$ is the distance from atom $i$ to neighbor $j$, $\phi(r)$ is a pair potential characterizing the core-core repulsion of the atomic nuclei, $\rho_{i}$ is the electron density at atom $i$, and $U(\rho)$ is the embedding energy due to the attraction between the nucleus and ambient electron density. Within the EAM approximation the electron density is also taken to have a pairwise form,

$$
\rho_{i}=\sum_{j} f\left(r_{i j}\right)
$$

The EAM offers a computationally tractable description of the material response which appears to describe many metals quite adequately. ${ }^{20}$

Two separate methodologies are employed to obtain the positions of the atomic neighbors of the representative atoms that are necessary for the evaluation of (3). The energy of representative atoms experiencing a slowly varying deformation in their vicinity is computed in a local fashion where it is assumed that the nearby environment of the atom is well-characterized by the deformation gradient at its position. This is essentially the continuum approximation, and this limit of the formulation corresponds to a nonlinear anisotropic elastic description of the material. At the other extreme are atoms experiencing large variations of deformation in their vicinity. These atoms are computed nonlocally in 
the sense that the positions of the neighboring atoms are independent of the deformation at the representative atom position. This corresponds to the lattice statics or atomistics limit of the formulation. ${ }^{14}$

The total energy of the system can now be computed from (2) and (3) and equilibrium configurations are identified by minimizing this energy with respect to the representative atom positions. This approach corresponds to a zero temperature quasistatic solution. The minimization is carried out by a quasi-Newton solver with a conjugate gradient backup when the initial guess is outside the basin of attraction of the Newton solver (see Ref. 17 for details). At each relaxed configuration the forces (per unit thickness in the out-of-plane direction) on the representative atoms are brought to below $10^{-6} \mathrm{eV} / \AA^{2}$.

A pseudo-two-dimensional implementation of quasicontinuum was used in the current study. Although all atomistic calculations were made in three dimensions (i.e., each representative atom is surrounded by a sphere of atoms for the purpose of calculating its energy), the displacement fields were constrained to have no variation in the out-of-plane $z$-direction, thus,

$$
u_{x}=u_{x}(x, y), \quad u_{y}=u_{y}(x, y), \quad u_{z}=u_{z}(x, y),
$$

where $u_{x}, u_{y}$, and $u_{z}$ are the displacements in the respective directions. This is a form of generalized plane strain.

Finally, the selection of representative atoms and their local versus nonlocal status is automatically carried out by the formulation using appropriate criteria and is constantly updated as the deformation progresses. More details on this and other fine points of the method can be found in the references cited at the beginning of this section.

\section{PROBLEM DESCRIPTION}

A rigid knife-like indenter is driven into a thin aluminum film $(0.1 \mu \mathrm{m}$ thick) resting on a rigid substrate. Both rectangular and cylindrical indenter cross sections were considered. Aluminum was chosen for these simulations, despite the fact that it is a difficult material to treat experimentally due to oxide formation, because of the availability of a good EAM potential for it. $^{21}$ EAM potentials traditionally suffer from serious underestimation of surface and stacking fault (SF) energies. However, the Ercolessi-Adams potential for $\mathrm{Al}$ has a SF energy of $0.10 \mathrm{~J} / \mathrm{m}^{2}$ which compares reasonably well with experimental values of $0.12-0.14 \mathrm{~J} / \mathrm{m}^{2}$ and a (111) surface energy of $0.87 \mathrm{~J} / \mathrm{m}^{2}$ which is again comparable with the experimental values of $1.14-1.20 \mathrm{~J} / \mathrm{m}^{2}$. The elastic moduli predicted by this potential are $C_{11}=117.74 \mathrm{GPa}, C_{12}=62.06 \mathrm{GPa}$, and $C_{44}=36.67 \mathrm{GPa}$. The experimental values extrapolated to $T=0$ are $C_{11}=118.0 \mathrm{GPa}, C_{12}=62.4 \mathrm{GPa}$, and $C_{44}=32.5 \mathrm{GPa}^{21}$ The equilibrium lattice constant is $a_{0}=4.032 \AA$.

In the following sections we will often make use of simple linear elastic solutions for an isotropic material to help rationalize the simulation results. The material parameters appearing in these solutions are the shear modulus $\mu$ and Poisson's ratio $\nu$. We compute effective values for these parameters from $C_{11}, C_{12}$, and $C_{44}$ by performing a Voigt average which ensures that the invariants of the elastic modulus tensor computed using the effective isotropic moduli are the same as those computed from the anisotropic moduli. ${ }^{22}$ This leads to the relations,

$$
\begin{gathered}
\mu=\frac{1}{5}\left(C_{11}-C_{12}+3 C_{44}\right), \\
\nu=\frac{1}{2}\left[\frac{C_{11}+4 C_{12}-2 C_{44}}{2 C_{11}+3 C_{12}+C_{44}}\right],
\end{gathered}
$$

which give $\mu=33.14 \mathrm{GPa}$ and $\nu=0.319$ for Ercolessi-Adams aluminum.

The knife-like geometry was dictated by the pseudo2D nature of the quasicontinuum model adopted. The prefix pseudo is meant to emphasize that although the analysis is carried out in a 2D coordinate system, outof-plane displacements are allowed and all atomistic calculations are three-dimensional, as explained in the previous section. Within this setting only dislocations with line directions perpendicular to the plane of analysis can be nucleated, and no variation in the out-of-plane $z$-direction can be sustained. These constraints appear to be compatible with the two-dimensional nature of the indenter, although some deformation paths may be unavailable.

Two different crystallographic orientations were investigated, as displayed in Fig. 1 where the dimension of the investigated system are also given. In the first configuration the film was oriented so that the preferred slip system $\langle 110\rangle\{111\}$ was parallel to the indentation direction to facilitate dislocation nucleation [see Fig. 1(a)] — this will be referred to as the dislocation orientation. In the second configuration the indenter was driven into a (111) surface of the crystal [see Fig. 1(b)]. The preferred slip system is now angled with respect to the indentation direction. In this case the indentation was accommodated by a deformation twinning mechanism, as will be seen in a following section, and so this configuration is referred to as the twinning orientation.

In addition to the two different crystallographic orientations, two different indenter geometries were studied. The first, as depicted in Fig. 1, is a rectangular indenter with a width of about $25 \AA$. The second indenter geometry (not depicted in the figures) was a cylindrical indenter with a radius of $11.6 \AA$. We note that the choice of indenter size was dictated by convenience and does 


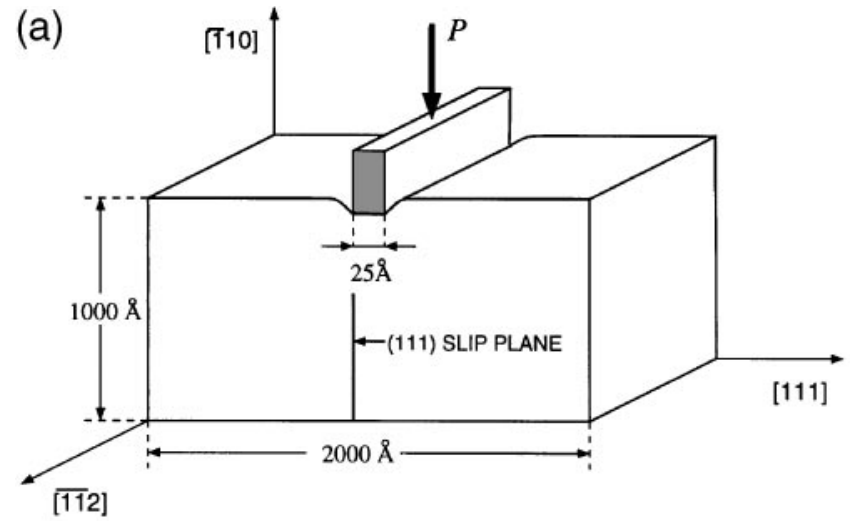

(b)

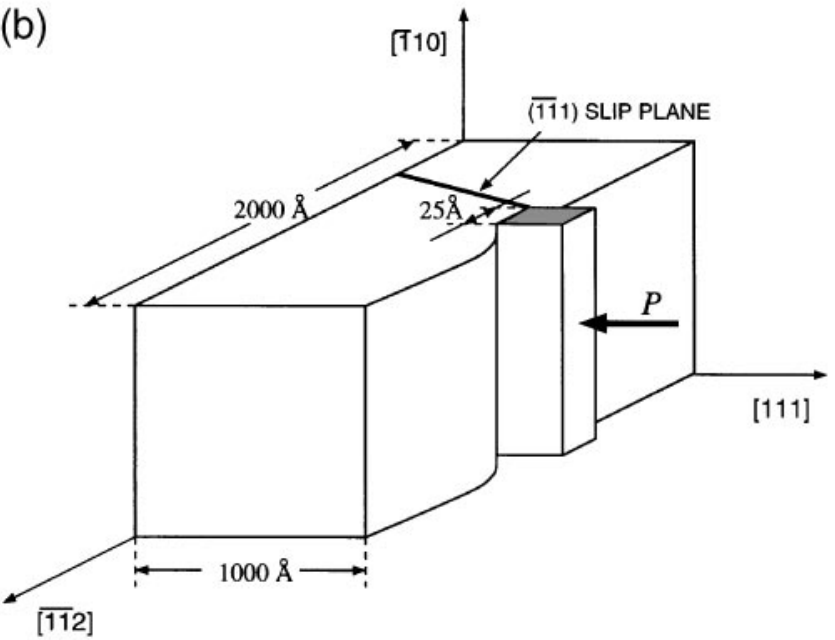

FIG. 1. Schematic representation of the nanoindentation model: (a) dislocation orientation and (b) twinning orientation.

not reflect a fundamental limitation of the system sizes that can be considered. For both indenter geometries the indenter was modeled as a displacement boundary condition applied to the surface atoms lying beneath it. The indenter is thus rigid and phantom in the sense that the interactions between tip atoms and film atoms are not considered. The substrate was modeled as a rigid surface allowing no displacements, and on the sides of the model symmetry boundary conditions were applied. The top surface of the film (aside from the region directly beneath the indenter) was left free. Between the indenter and the crystal, both friction-free and perfect stick conditions were considered. Neither boundary condition reproduces the complexities of interaction between the tip and the crystal; however, they may be expected to act as bounds to this behavior. In practice, the results were not greatly influenced by the choice of boundary condition.

The thin film investigated in this simulation is $0.1 \mu \mathrm{m}$ thick, $0.2 \mu \mathrm{m}$ wide, and infinite in the out-ofplane direction, as indicated above. This system size was selected to ensure that far-field boundary conditions did not affect the behavior in the vicinity of the indenter. Increasing system size did not change the results. The system investigated here is very large by current atomistic modeling standards. A standard lattice statics analysis of this system would require the treatment of 1.3 million atoms or about 4 million degrees of freedom and would have to be performed on a parallel supercomputer. By using the quasicontinuum method the computational intensity is greatly reduced. Within the quasicontinuum calculation, at most only 4000 atoms are treated explicitly (12,000 degrees of freedom), and a simulation can be run on a desktop workstation in a few days.

\section{NANOINDENTATION IN THE DISLOCATION ORIENTATION}

\section{A. Rectangular indenter}

The first orientation investigated was selected, as explained, to facilitate dislocation emission by orienting the crystal such that the indentation direction coincides with a preferred slip direction [110] and the indenter sides are parallel to the (111) slip planes. The indenter is thus pushed into a $(\overline{1} 10)$ plane of the crystal. The indenter is a rigid rectangular block $23.3 \AA$ wide (10 lattice spacings in the $x$-direction). Friction-free conditions were assumed between the indenter and the thin film surface. The number of atoms in contact with the indenter remains constant (the atoms directly beneath the indenter displace very little in the $x$-direction and it is assumed that they cannot slip out from under the indenter).

\section{Load-displacement response}

The load-displacement curve obtained from the simulation is presented in Fig. 2. The calculations were

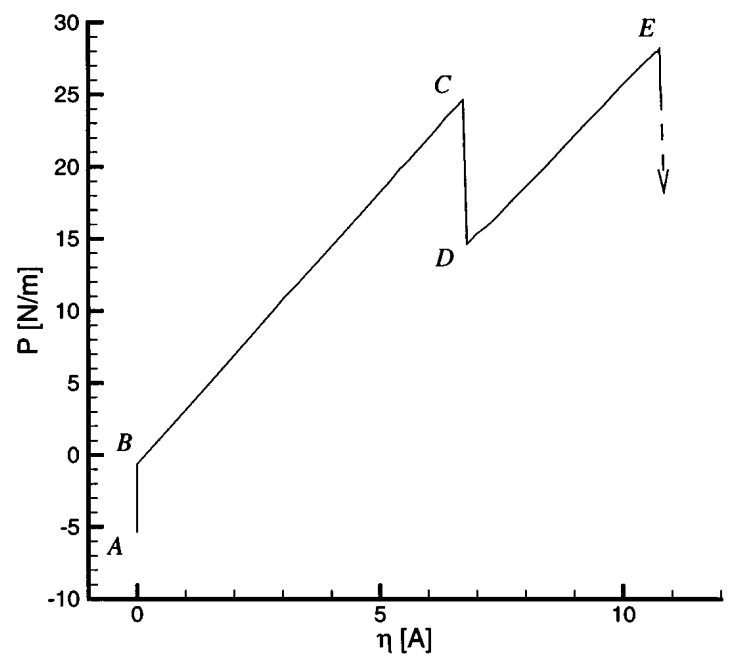

FIG. 2. Computed load versus displacement curve for nanoindentation into a (110) plane (dislocation orientation) of an aluminum thin film with a rigid rectangular indenter. 
carried out with displacement control and thus the figure represents the load required to hold the indenter at a given indentation depth. At each load step the indentation depth is increased by $0.1 \AA$. Loads are given in Newtons per meter length of the indenter in the outof-plane direction. The curve starts out negative at a load of $-5.40 \mathrm{~N} / \mathrm{m}$ (point $A$ ) and climbs to $-0.63 \mathrm{~N} / \mathrm{m}$ (point $B$ ) at zero indentation. This corresponds to the initial surface relaxation in the vicinity of the indenter. The load is still negative at point $B$ because the atoms beneath the indenter are artificially held at zero displacement instead of being allowed to displace slightly downward with the rest of the surface.

Following this initial relaxation the response is linear as predicted by elasticity theory for a rigid rectangular indenter which maintains a constant contact area with varying indentation depth. ${ }^{23}$ While the elasticity solution predicts a linear response, it is noncommittal with respect to the slope, and therefore it cannot simply be used as a test for the accuracy of the computations in the linear regime. The reason for this is that the elasticity solution is computed for the idealized case of rigid indentation into an elastic half-space. The use of an infinite domain leads to an arbitrary unresolved constant in the elastic displacement fields which finds its way into the slope of the load-displacement curve.

\section{Dislocation nucleation}

The linear portion of the load-displacement curve terminates abruptly at $C$ with the emission of dislocations from beneath the indenter. The dislocations nucleate in a single minimization step, going from incipient slip distributions at the indenter tips to a fully formed dipole of dissociated edge dislocations beneath the surface. The dislocations are emitted at an indentation depth of $6.7 \AA$ at a load per unit thickness of $24.7 \mathrm{~N} / \mathrm{m}$ which corresponds to a hardness (i.e., mean pressure) of $9.8 \mathrm{GPa}$. This is more than two orders of magnitude larger than the values reported in macroscopic hardness tests of aluminum, which are about $40 \mathrm{MPa}^{3}$ We may also compare this value with recent nanoindentation experiments in (100) single crystal aluminum ${ }^{24}$ which measured a peak hardness of $1.1 \mathrm{GPa}$ using a Berkovich indenter with a $0.1 \mu \mathrm{m}$ tip radius. This is consistent with many observations of significant increases in hardness with reduced indentation size (see, for example, Ref. 25).

The dislocated structure beneath the indenter is given in Fig. 3 along with the out-of-plane displacements experienced by the atoms. Two dissociated $\langle 110\rangle$ edge dislocations have been emitted beneath the indenter tips, first from the right and then from the left. In the simulation the symmetry was broken due to numerical noise. This is acceptable because it is expected that

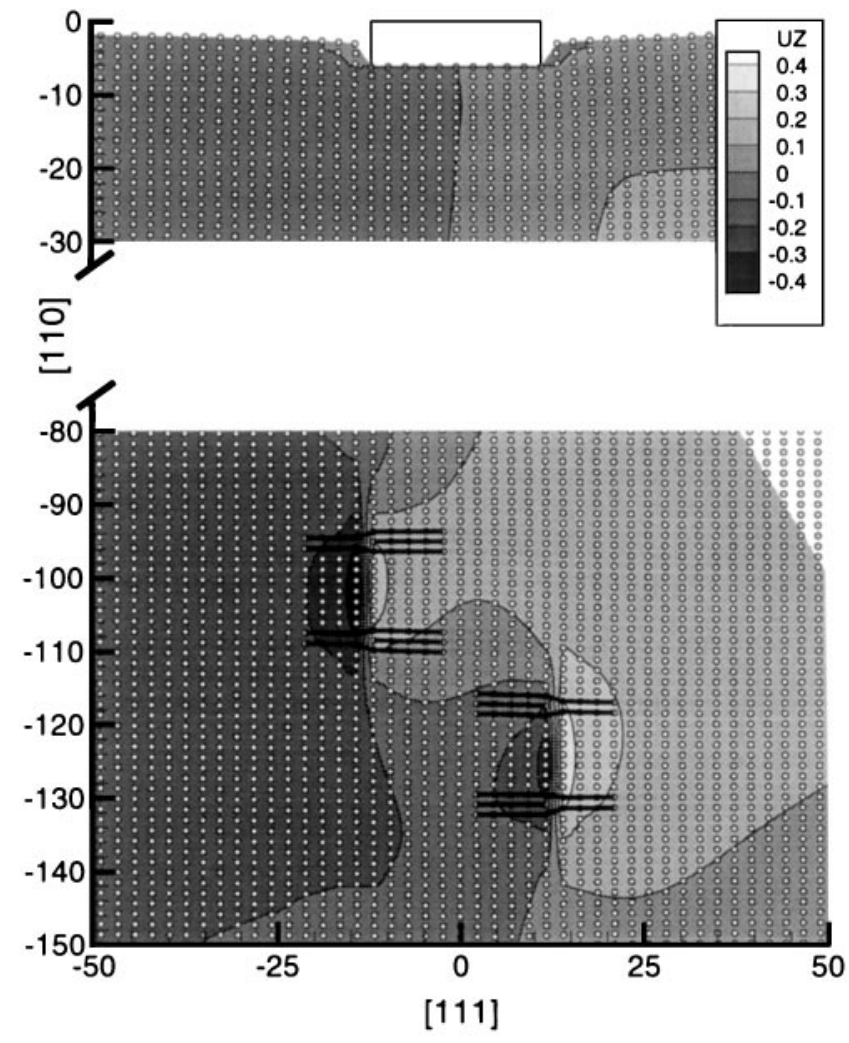

FIG. 3. Dislocated structure beneath the rectangular indenter and corresponding out-of-plane displacement plot after nucleation (dimensions and displacements in $\AA$ ).

symmetry will also be broken in real systems due to imperfections and misalignments.

The dislocations are composed of $1 / 6\langle 112\rangle$ Shockley partials which bound a stacking fault. On the left,

$$
\begin{array}{r}
\frac{1}{2}[\overline{1} 10] \rightarrow \frac{1}{6}[\overline{1} 2 \overline{1}]+\frac{1}{6}[\overline{2} 11], \\
(\text { top }) \quad(\text { bottom })
\end{array}
$$

and on the right,

$$
\begin{array}{r}
\frac{1}{2}[1 \overline{1} 0] \rightarrow \frac{1}{6}[1 \overline{2} 1]+\frac{1}{6}[2 \overline{1} \overline{1}] . \\
(\text { top }) \quad(\text { bottom })
\end{array}
$$

Isolated Shockley partials carry an out-of-plane component of $\sqrt{6} a_{0} / 12$ where $a_{0}$ is the lattice parameter. For aluminum this yields a value of $0.82 \AA$. In the simulations, a smaller relative out-of-plane displacement was observed across the stacking fault of $0.65 \AA$. This is most likely due to the small splitting distance $(\sim 13.5 \AA)$ and the resulting core-core interactions of the partials. This splitting distance is smaller than that of an isolated edge dislocation which was found to be $15.4 \AA^{13}$ as a result of the dipole configuration the dislocations occupy. It should be noted that both values are large compared to the experimental bounds set by Mills and Stadelmann ${ }^{26}$ 
probably as a result of the relatively low stacking fault energy of the EAM potential.

The two nucleated dislocations adopt a staggered configuration instead of arranging so that their extra half-planes line up. This is well known from elementary dislocation theory. Consider a simple model where we neglect the stress field due to the indenter, the image effects due to the surface, and the dissociation of the dislocations into partials. We then have an edge dislocation dipole of strength $b$ and width $2 a$ (see Fig. 4). The dipole energy as a function of separation $d$ is given by, ${ }^{27}$

$$
U_{d}=\frac{\mu b^{2}}{2 \pi(1-\nu)}\left[\ln \frac{r}{b}-\frac{1}{2} \cos 2 \theta\right],
$$

where the polar coordinates $(r, \theta)$ fix the relative positions of the two dislocation lines. For the given configuration, $r=\sqrt{4 a^{2}+d^{2}}$ and $\theta=\tan ^{-1}(2 a / d)$. The dipole energy (10) has a minimum value at $\theta=\pi / 4$ which corresponds to a position of stable equilibrium at a distance of $d=2 a=23.2 \AA$. The case where the dislocations line up at $\theta=0$ corresponds to a local maximum of the energy or to a position of unstable equilibrium. In the simulation, the distance between the centers of the dissociated dislocations is $22.2 \AA$, in good agreement with the predicted value. A more elaborate analysis which accounts for the dissociation of the dislocations into partials and makes use of a more general expression for the interaction energies of dislocations of arbitrary Burgers vectors resulted in a similar solution and is not presented here for reasons of brevity.

After nucleation, the dislocation dipole travels into the bulk and its center settles at a depth of $355 \AA$. It is believed that this value is independent of the simulated system size; however, simulations with larger models were not carried out to verify this. Figure 5 shows a closeup of the mesh near the indenter after the dipole

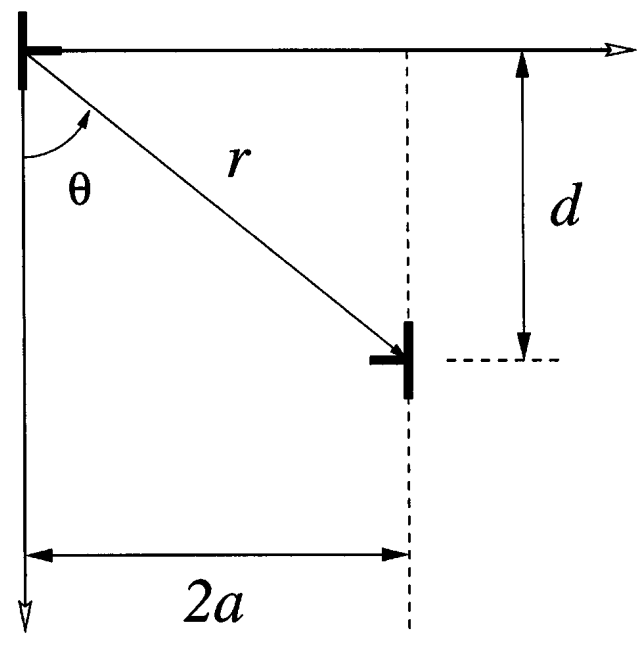

FIG. 4. Simple dislocation dipole model. settles at its equilibrium depth. The mesh is fully refined (i.e., all atoms are represented) near the indenter, where surface effects come into play, and near the dislocation cores. Away from these nonlocal regions, the mesh is coarsened out, resulting in significant reductions in the number of degrees of freedom that must be treated. In order to obtain a mesh, such as the one in Fig. 5, where only the regions in the immediate vicinity of defects are fully refined, it was necessary to modify the criterion selecting between local and nonlocal methodologies (see Ref. 17). Instead of using the total deformation gradient $\mathbf{F}$ in the criterion, $\mathbf{F}$ is decomposed into its plastic and elastic parts, $\mathbf{F}=\mathbf{F}^{e} \mathbf{F}^{p}$, and only the elastic part is used in the criterion. This will be explained in more detail in a forthcoming publication.

\section{Estimation of the Peierls stress}

We may use the equilibrium distance to obtain an estimate for the Peierls stress predicted by the EAM potential. Neglecting the dissociation of the dislocations into partials, we consider an edge dislocation dipole at depth $h$ beneath the indenter. For simplicity, we take the dipole to be lined up and not staggered. The dipole is free to glide along the indentation direction and we seek the equilibrium distance at which the forces in this direction cancel out. The forces between the dislocations making up the dipole are zero and so we focus on a single dislocation. Aside from lattice friction, there are two forces acting on this dislocation, (i) the Peach-Koehler force $\left(F_{\mathrm{PK}}\right)$ due to the indenter stress field driving the dislocation into the bulk, and (ii) the image force $\left(F_{I}\right)$

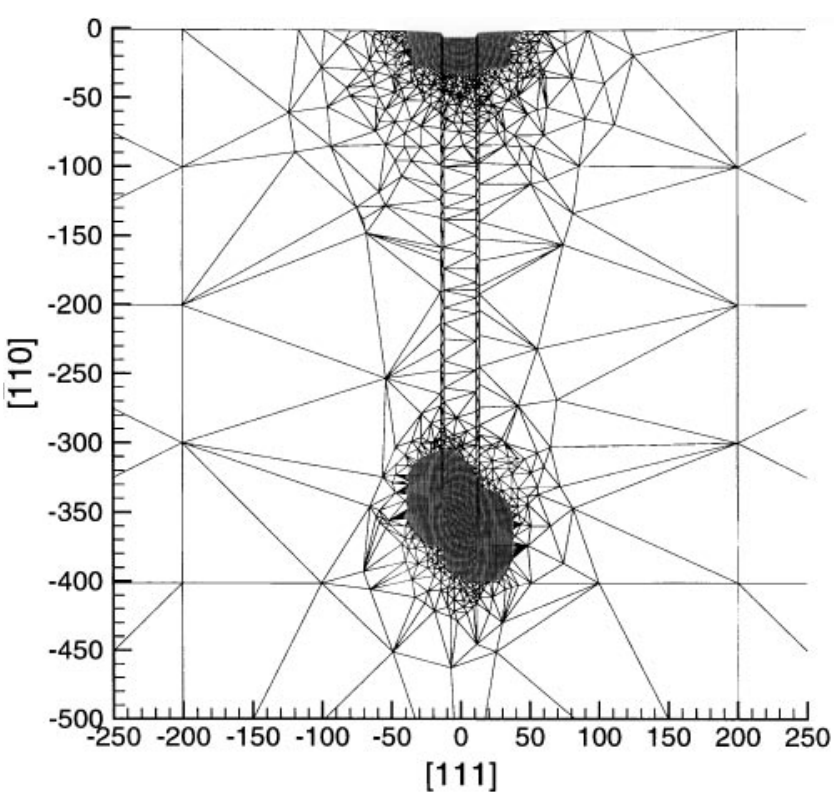

FIG. 5. Quasicontinuum mesh near the indenter after dislocation emission. The nodal positions correspond to representative atom sites. Dimensions are in $\AA$. 
pulling the dislocation to the surface. The force on the dislocation is the sum of these forces. The dislocation is attracted to the surface, very near to it, and repulsed everywhere else. Upon emission, the dislocation escapes the attractive region and propagates into the bulk. It will continue until it is stopped by lattice friction. Thus at the equilibrium depth, the force on the dislocation will be balanced by the lattice friction force resulting from the Peierls stress $\sigma_{p}$ (i.e., the stress required to move a dislocation in a lattice),

$$
F_{\mathrm{PK}}+F_{I}=b \sigma_{p} .
$$

To compute the Peach-Koehler force, we require the shear stress field beneath the indenter. For a frictionless rectangular indenter of width $2 a$ acting on an elastic body occupying the lower half-plane, $y<0$, the shear stress in bipolar coordinates is, ${ }^{23}$

$$
\sigma_{x y}=-\frac{P r^{2} \sin \theta}{\pi\left(r_{1} r_{2}\right)^{3 / 2}} \sin \left[\theta-\frac{3}{2}\left(\theta_{1}+\theta_{2}\right)\right],
$$

where $P$ is the indentation load and the coordinate system is defined in Fig. 6. At a distance $h$ beneath the right indenter tip we have, $r=\sqrt{a^{2}+h^{2}}, r_{1}=h, r_{2}=$ $\sqrt{4 a^{2}+h^{2}}, \theta=-\tan ^{-1} h / a, \theta_{1}=-\pi / 2$, and $\theta_{2}=$ $-\tan ^{-1}(h / 2 a)$. The resulting Peach-Koehler force is,

$$
F_{\mathrm{PK}}(h)=(\mathbf{b} \cdot \boldsymbol{\sigma}) \times \ell=b \sigma_{x y}(h),
$$

where $\mathbf{b}$ is the Burgers vector, $\boldsymbol{\sigma}$ is the applied stress tensor, and $\ell$ is the dislocation line vector.

The image force acting on one of the dislocations of a dipole of width $d$ at depth $h$ beneath the surface can be shown to be,

$$
F_{I}=\frac{\mu b^{2}}{\pi(1-\nu)}\left[\frac{1}{4 h}-\frac{4 h^{3}\left(4 h^{2}-3 d^{2}\right)}{\left(4 h^{2}+d^{2}\right)^{3}}\right] .
$$

The left term in the square brackets represents the attraction of the dislocation to its image. The right term corresponds to the repulsion from the image of the other dislocation in the dipole and the contribution of an additional stress field added to obtain correct boundary conditions on the free surface. ${ }^{22}$ Using the equilibrium depth of $h=355 \AA$ and the load after emission,

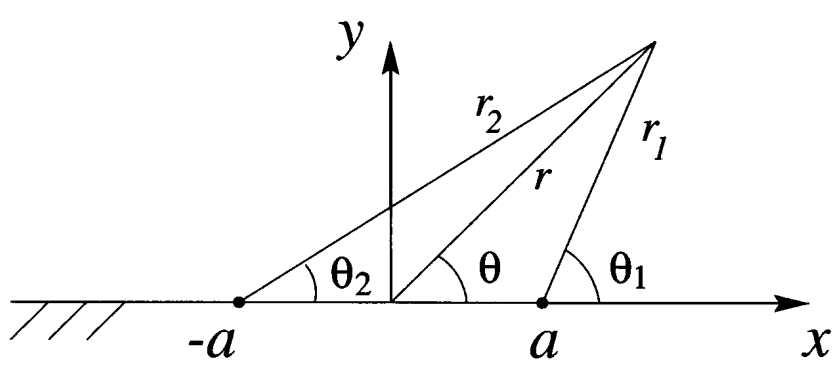

FIG. 6. Bipolar coordinates for a $2 a$ indentation contact. which was $14.7 \mathrm{~N} / \mathrm{m}$, we obtain a Peierls stress, $\sigma_{p} \simeq$ $8.3 \mathrm{MPa}$ or $\sigma_{p} \simeq 2.5 \times 10^{-4} \mu$. This compares to a value of $6.8 \times 10^{-4} \mu$, obtained previously by Shenoy et $a{ }^{17}{ }^{17}$ for a screw dislocation using the same EAM potential. Experimental estimates for the Peierls stress in aluminum vary from $2.7 \times 10^{-5} \mu$ to about $10^{-3} \mu .^{28}$ The lower estimate, recently obtained by Kosugi and Kino, ${ }^{28}$ is presented by the authors as being more in line with yield stress measurements and other observations. If this is true, then the Ercolessi-Adams potential appears to overestimate the Peierls stress by about an order of magnitude.

The shear stress distribution beneath the indenter immediately prior to indentation is given in Fig. 7. The stresses presented in the figure are the atomic level stresses computed for each atom (see, for example, Refs. 29 and 30). We focus on the $x y$ component of the stress tensor because for this orientation this is also the resolved shear stress on the active slip system which controls dislocation emission. The maximum shear stress prior to emission is $3.0 \mathrm{GPa}$ or about $\mu / 10$ which is of the order of the theoretical elastic limit. We note that the maximum shear stress lies very close to the surface where the atomic level stresses, which are rigorously defined only in bulk regions, are suspect and thus the maximum stress must be accepted with reservation. The shear stress computed from elasticity theory in Eq. (12) predicts an infinite stress at the indenter tip, so direct comparison is not possible. The maximum computed shear strain is $10.3 \%$.

\section{Elastic model for dislocation emission}

It is interesting to examine how well a simple elastic model can predict the load necessary for dislocation emission. Since elasticity theory predicts an infinite stress at the indenter tip, a criterion based on critical resolved shear stress cannot be applied here and instead we turn to an energetic criterion. Consider the energy balance of the nucleation $C \rightarrow D$ (Fig. 2). Prior to nucleation the total energy is,

$$
U_{C}=\frac{1}{2} P_{c r} \eta_{c r}=\frac{1}{2} k \eta_{c r}^{2},
$$

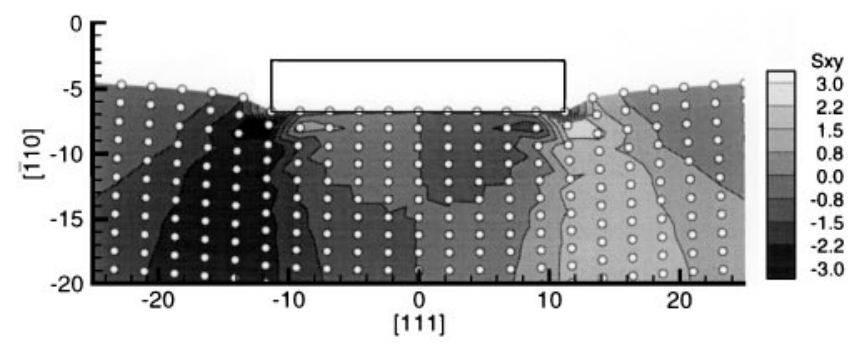

FIG. 7. Shear stress distribution beneath the rectangular indenter (dislocation orientation) immediately prior to dislocation emission (spatial dimensions are in $\AA$, stress is in GPa). 
where $P_{c r}$ is the maximum load at emission, $\eta_{c r}$ is the indentation depth at emission, and $k$ is the initial linear slope of the load-displacement curve. After nucleation it is as though a Burgers vector has been removed from the elastic loading, thus the force is reduced from the critical load to $\left(1-b / \eta_{c r}\right) P_{c r}$ and the elastic indentation depth is reduced to $\eta_{c r}-b$. The total energy at $D$ is then,

$$
U_{D}=\frac{1}{2} k\left(\eta_{c r}-b\right)^{2}+U_{\perp},
$$

where $U_{\perp}$ is the additional energy due to the presence of the dislocations. Equating (15) and (16) and rearranging we have,

$$
P_{c r}=\frac{U_{\perp}}{b}+\frac{1}{2} k b .
$$

If we neglect the interaction between the emitted dislocations and the stress field of the indenter, we may approximate $U_{\perp}$ as the energy of the dislocation dipole (seen in Fig. 4) when positioned at its equilibrium depth $h$ beneath the surface as computed from (11). The energy will be composed of three parts: (1) the elastic energy including image effects, ${ }^{27}$

$$
U_{I}=\frac{\mu b^{2}}{4 \pi(1-\nu)}\left[\ln \frac{2 h}{b}+\ln \frac{2(h+d)}{b}\right] ;
$$

(2) the dipole interaction energy (10) at the equilibrium distance $d=2 a$ (i.e., $r=2 \sqrt{2} a$ and $\theta=45^{\circ}$ ),

$$
U_{d}^{\min }=\frac{\mu b^{2}}{2 \pi(1-\nu)} \ln \frac{2 \sqrt{2} a}{b} ;
$$

and (3) the additional surface energy due to the formation of steps on the surface,

$$
U_{\gamma}=2 \gamma_{111} b,
$$

where $\gamma_{111}$ is the (111) surface energy. Summing (18)(20) and substituting into (17) we obtain the elastic estimate for the critical load for dislocation emission,

$$
\begin{aligned}
P_{c r}= & \frac{\mu_{b}}{4 \pi(1-\nu)} \ln \frac{32 h(h+2 a) a^{2}}{b^{4}} \\
& +2 \gamma_{111}+\frac{1}{2} k b .
\end{aligned}
$$

Substituting in the appropriate values including $\gamma_{111}=$ $0.869 \mathrm{~J} / \mathrm{m}^{2}$ and $k=36.7 \mathrm{GPa}$, we find $P_{c r}=24.6 \mathrm{~N} / \mathrm{m}$ (for the equilibrium depth $h=355 \AA$ ) in surprisingly close agreement with the observed value of $24.7 \mathrm{~N} / \mathrm{m}$.

Following nucleation a significant drop in load is observed. The elastic estimate consistent with the above model for this load drop is that it corresponds to relaxation of a Burgers vector worth of elastic indentation. Thus,

$$
\Delta P=k b,
$$

which gives $\Delta P=10.5 \mathrm{~N} / \mathrm{m}$. The actual load drop observed is $10.0 \mathrm{~N} / \mathrm{m}$.

\section{Second dislocation emission}

After the load drop, the load-displacement curve picks up and resumes a linear ascent with a slightly more moderate slope of $34.4 \mathrm{GPa}$ (as compared with the initial slope of $36.7 \mathrm{GPa}$ ). The change in slope is a result of the presence of the emitted dipole which modifies the compliance of the elastic medium, making it a little less stiff. A second dislocation dipole is emitted at an indentation depth of $10.8 \AA$ at a load of $28.3 \mathrm{~N} / \mathrm{m}$ (point $E$ in Fig. 2). It is interesting that the second emission occurred at a higher load than the first, again as a result of the presence of the emitted dipole. The simulation was terminated at this point without an attempt to establish the new equilibrium depths of the dislocations, thus the load drop associated with the second emission was not obtained. We investigate unloading in the next section for a cylindrical indenter.

\section{Density of geometrically necessary dislocations}

A connection can be made here with the simple model for the density of geometrically necessary dislocations beneath an indenter proposed by François et al. ${ }^{31}$ Neglecting any elastic deformation, the model assumes that a new dislocation dipole is punched out every time the indenter travels a Burgers vector $b(2.85 \AA$ for aluminum); the resulting density is,

$$
\rho_{G N D}=\frac{2 n}{A}=\frac{2 \eta}{b A},
$$

where $n$ is the number of dislocations nucleated, $A$ is the area of crystal projected on the $x y$-plane, and $\eta$ is the indentation depth. In the simulation the model assumptions are not satisfied. The second emission occurs $4.1 \AA$ after the first, which is about $1.4 b$. If we assume that subsequent emission will occur at similar intervals, this leads to a dislocation density about $30 \%$ lower than that predicted by the model. The discrepancy appears to be related to the backstress exerted by the dislocation dipole which was emitted earlier, an effect which is neglected by François' model. Such an assumption may be appropriate in the case of other dislocation sources, such as cracks, where emitted dislocations are driven to large distances by the crack tip stress field, creating dislocation free zones of the order of thousands of angstroms. However, the indenter stress field which decays as $1 / r$ (as opposed to $1 / \sqrt{r}$ for the crack) keeps the dislocations far closer and thus heightens their effect on subsequent emission. An expression for the density of geometrically necessary dislocations beneath a conical indenter has recently been given by $\mathrm{Nix}^{32}$

\section{Boundary condition effects}

Finally, a note on the effect of boundary conditions on the simulation outcome. The results presented in this 
section are for a friction-free indenter. The same simulation was also carried out for perfect-stick boundary conditions. In both cases the qualitative response was the same and the quantitative differences were relatively small. The main difference was that emission occurred a little earlier in the perfect stick simulation $(6.0 \AA$ A instead of $6.7 \AA)$ and at a slightly lower load $(22.1 \mathrm{~N} / \mathrm{m}$ instead of $24.7 \mathrm{~N} / \mathrm{m}$ ). The resolved shear stress at emission was a little higher (3.7 GPa compared with $3.0 \mathrm{GPa}$ ). Thus, it appears that the indenter boundary condition is of limited significance. In the remaining simulations, perfect-stick conditions were employed because they are computationally more convenient.

\section{B. Cylindrical indenter}

We now replace the rectangular indenter with a cylindrical indenter and repeat the indentation simulation. The new indenter has a radius equal to the rectangular indenter half-width, $R=a=11.64 \AA$. The crystallographic orientation of the thin film is the same as in the previous section and as depicted in Fig. 1(a).

Perfect-stick conditions were used to characterize the contact between the indenter and the film surface (as motivated in the concluding remarks of the previous section). For a cylindrical indenter, the contact area increases with indentation depth and thus new atoms will occasionally come into contact with the indenter surface. To account for this the boundary conditions for the cylindrical indenter are handled as follows: at every load step the indenter is pushed a small distance $(0.2 \AA)$ farther into the crystal. All atoms already in contact with the indenter are moved down with it. In addition, any atoms that are found "inside" the indenter after it is repositioned are relocated onto its surface and constrained to remain there. The energy of the new configuration is then minimized. At the end, any atoms that are found to be held to the indenter face by a tensile load are released and allowed to move away as the energy is minimized once more. This proved to be important during the nucleation phase.

\section{Load-displacement response}

The load-displacement curve for the cylindrical indenter including the loading phase, nucleation phase, and unloading phase is presented in Fig. 8. As for the rectangular indenter, it is not possible to obtain a definitive relationship between load and indentation depth from the elasticity theory solution for indentation into a semi-infinite half-plane. Instead, the theory predicts the relationship between indentation load and the contact half-width $a,{ }^{23}$

$$
P=\frac{\pi \mu a^{2}}{2(1-\nu) R}
$$

We use the solution for a frictionless punch for convenience. The elastic solution for perfect-stick conditions is analytically more complex than the friction-free case, while at the same time both solutions are quantitatively very similar except in the immediate vicinity of the indenter. Substituting in the appropriate values $(\mu=$ $33.14 \mathrm{GPa}, \nu=0.319$, and $R=11.64 \AA$ ) the relation reduces to $P=0.657 a^{2}$ with $P$ obtained in $\mathrm{N} / \mathrm{m}$ and $a$ taken in $\AA$.

In elasticity theory it is assumed that the contact $a$ grows continuously with the load $P$; however, due to the atomic resolution of our simulation the contact area grows in quantized jumps as new atoms become trapped by the indenter. Figure 9 compares the load versus contact area obtained from the simulation compared with that predicted by (24). We see that up to dislocation emission the elastic curve (24) nicely characterizes the atomic solution that follows it in stepwise fashion.

Despite the sudden increases in contact area the load-displacement curve in Fig. 8 is very smooth. Points $A$ and $B$ correspond to load steps where the contact area increased due to the trapping of new surface atoms by the indenter. Between these points the response is linear with slight changes of slope occurring with the trapping of new atoms, as might be expected. However, the curve deviates from the linear response as it approaches the point of dislocation nucleation at $C$. Overall, the response has a power law form as would be expected for an indenter of varying cross section.

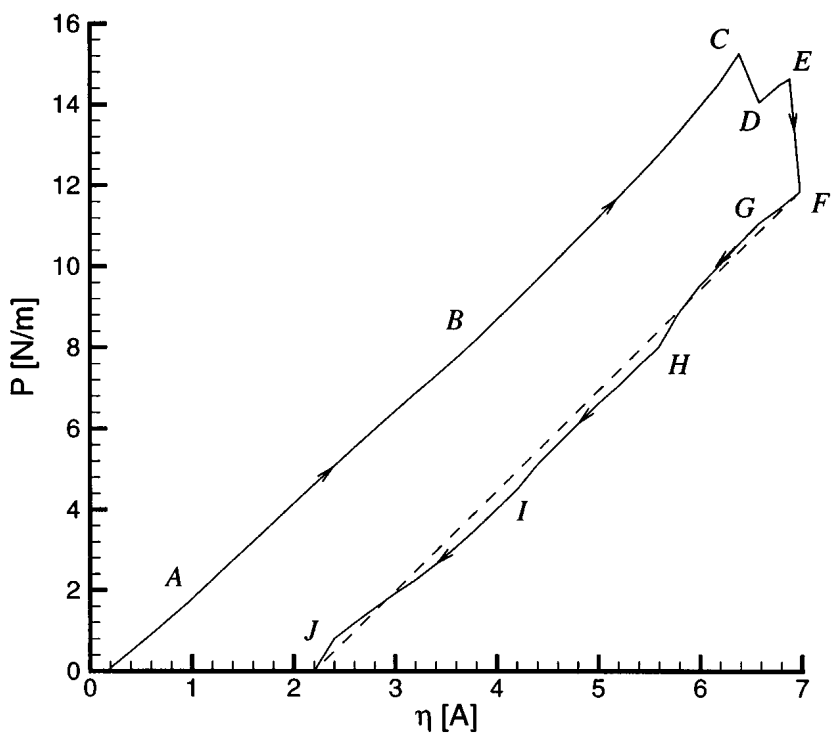

FIG. 8. Computed load versus displacement curve for nanoindentation into a (110) plane (dislocation orientation) of an aluminum thin film with a rigid cylindrical indenter. 


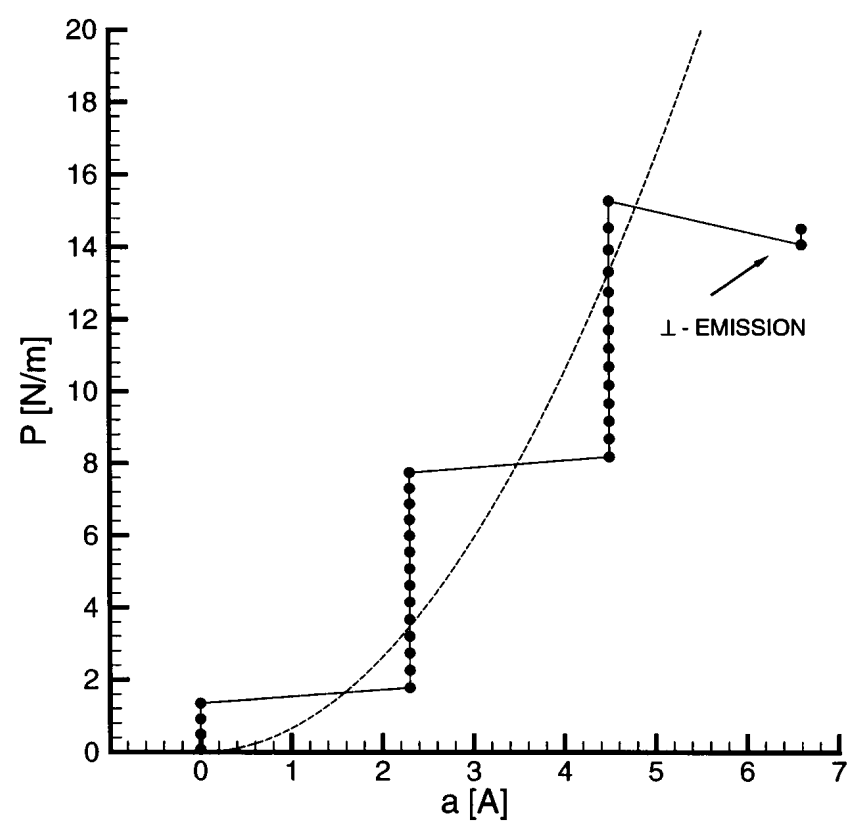

FIG. 9. Load versus contact half-width for cylindrical indentation in the dislocation orientation (points correspond to simulation results and dashed line to the elasticity theory prediction).

\section{Dislocation nucleation}

The elastic response continues up to an indentation of $6.6 \AA$ when a dipole of Shockley partial dislocations is emitted (see Fig. 10). Immediately prior to nucleation, five atoms (per repeat distance in the out-of-plane direction) are in contact with the indenter. Dislocation nucleation occurs by the atoms originally just outside the contact region (marked A and B in Fig. 10) moving up to the indenter and those at the original outer contact points (marked $\mathrm{A}^{\prime}$ and $\mathrm{B}^{\prime}$ in the figure) moving slightly down and away from the indenter. In this way surface steps are formed beneath the indenter, as indicated in the figure. The reason for the emission of Shockley partials and not full edge dislocations is the interference of the indenter with the movement of atoms A and B. If the boundary conditions are modified so that these atoms are allowed to move "through" the indenter without penalty, a dipole of fully dissociated edge dislocations is emitted at this point just as observed for the rectangular indenter. However, with the indenter present as a rigid barrier, atoms $\mathrm{A}$ and $\mathrm{B}$ move up, come in contact with the indenter and stop there, and the crystal has to make do with partials.

The emission occurs at a load of $15.3 \mathrm{~N} / \mathrm{m}(25 \%$ lower than the nucleation load observed for the perfectstick rectangular indenter) and a corresponding shear stress of $3.8 \mathrm{GPa}$ (close to the $3.7 \mathrm{GPa}$ observed for that case). The partials move down and away from the indenter laying down a stacking fault in their wake and until settling at an equilibrium distance of $42 \AA$ below the surface. The partials are of type $\pm 1 / 6[\overline{2} 11]$, the

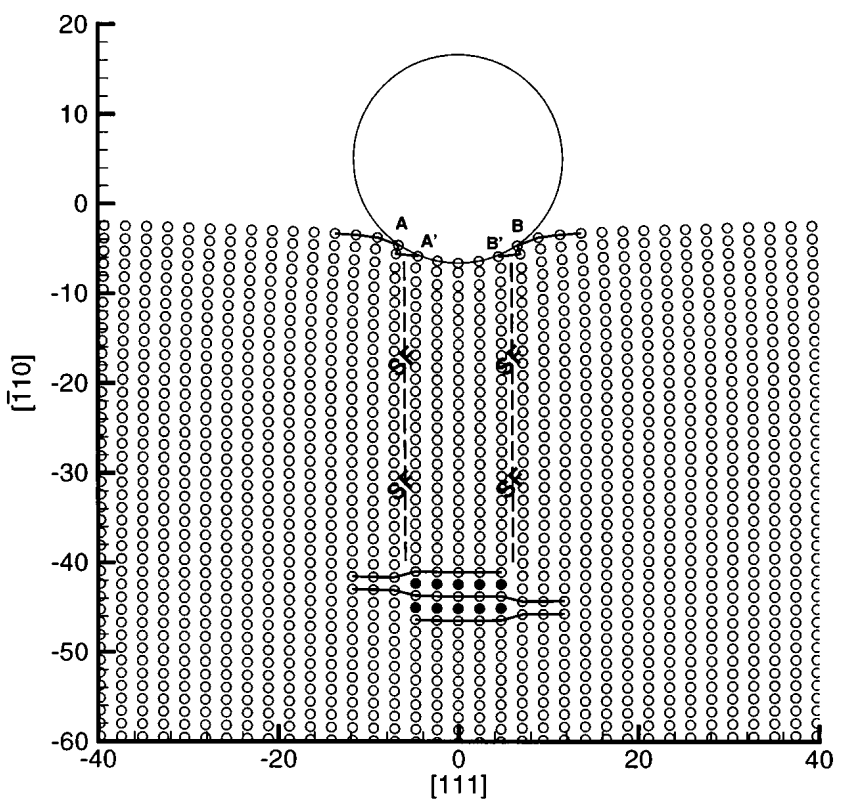

FIG. 10. Atomic structure beneath the cylindrical indenter at an indentation depth of $6.6 \AA$ showing the nucleated Shockley dipole and stacking fault (dimensions are in $\AA$ ).

same as the leading partials observed in the rectangular indentation. The dipole has a width of $9.5 \AA$, significantly smaller than the dipole width in the rectangular indenter which was simply equal to the indenter width of $23.2 \AA$. Another difference is that in the previous indentation a dipole of fully dissociated edge dislocations was nucleated, not Shockley partials as seen here.

These differences are due to the interaction and interference of the indenter geometry with the response of the underlying crystal. For the rectangular indenter the shear stress gradually builds up at the indenter tips (which remain in a constant position relative to the crystal) until emission occurs. However, for the cylindrical indenter, the indenter "tips," or outer points of contact, occasionally move out as more atoms come in contact. The maximum shear stress always lies close to these outer contact points. Thus the incipient slip buildup is constantly moving out as new atoms come into contact until the slip distribution becomes unstable and emission occurs.

The partial dislocations form a dipole which as in the case of the edge dipole discussed earlier, does not line up, although in this case the degree of misalignment is much smaller (see Fig. 10). This misalignment can be more clearly (and quantitatively) seen in Fig. 11, which shows the slip under the left and right contact points of the indenter immediately prior to emission and immediately after. Before emission the incipient slip profiles on the left and right are identical and overlap. However, after emission we see that the slip profiles are displaced relative to each other by a small amount 
(about $2 \AA$ ), indicating that the dislocation cores are not lined up.

The misalignment can be explained in terms of the same kind of simple model used earlier and presented in Fig. 4. The interaction energy for a dipole of Shockley partial dislocations can be shown to be,

$$
\begin{aligned}
U_{d}= & \frac{\mu b_{s}^{2}}{8 \pi(1-\nu)} \\
& \times\left[(4-\nu) \ln \frac{\sqrt{4 a^{2}+d^{2}}}{b_{s}}-\frac{3 d^{2}}{4 a^{2}+d^{2}}\right] \\
& +d \gamma_{\mathrm{SF}},
\end{aligned}
$$

where $b_{s}=a_{0} / \sqrt{6}$ is the magnitude of the Shockley partial Burgers vector. This energy is different from the dipole energy given in (10) which was evaluated for a dipole of pure edges. The energy given here accounts for both the edge and screw components of the partials and includes the additional stacking fault energy due to the misalignment. The inclusion of the stacking fault energy makes a large difference in the predicted equilibrium configuration. Without the stacking fault term, the dipole energy is minimum at,

$$
d=2 a \sqrt{\frac{2+\nu}{4-\nu}} .
$$

However, with increasing stacking fault energy, the equilibrium distance between partials decreases, and for aluminum $\left(\gamma_{\mathrm{SF}}=0.1 \mathrm{~J} / \mathrm{m}^{2}\right)$ the minimum is at zero, thus no misalignment is expected. This is essentially in agreement with the observed structure, since the small

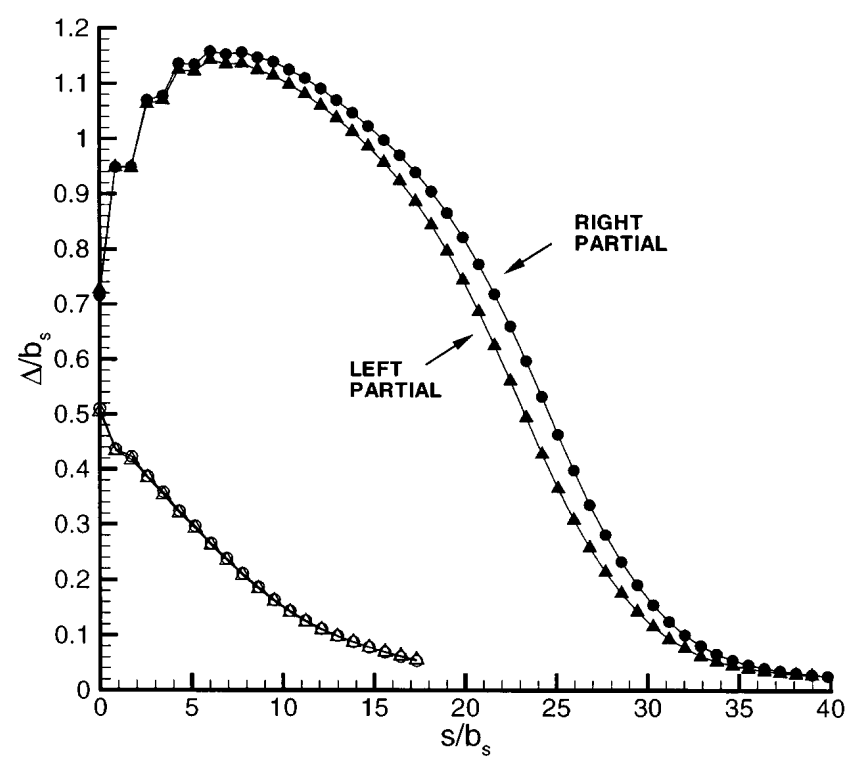

FIG. 11. Slip beneath the left $(\boldsymbol{\Delta})$ and right $(\mathbf{O})$ contact points of a cylindrical indenter, prior to emission (hollow symbols) and after emission (solid symbols). misalignment observed is beyond the resolution of the elastic model.

After emission of the partial dislocation dipole, the maximum stress normal to the indenter face $\left(\sigma_{y y}\right)$ is reduced from -29.4 to $-22.6 \mathrm{GPa}$ (which explains the drop in load upon emission). At the same time the shear stress beneath the indenter outer contact points increases from 3.8 to $4.5 \mathrm{GPa}$ and stays about constant until a second partial dislocation dipole is emitted at an indentation depth of $7 \AA$ and a load of $14.7 \mathrm{~N} / \mathrm{m}$ ( $E \rightarrow F$ in Fig. 8). The two dipoles reconstruct as a dissociated edge dislocation dipole very similar to the one observed earlier for the rectangular indenter. The dipoles in this case also take on a staggered configuration and travel downward into the bulk.

It is interesting to consider the atomistic mechanism for the emission of the second partial dipole considering the interference of the indenter with the formation of surface steps. Figure 12 shows the atomic structure beneath the indenter after emission of the second dipole. With the emission of the second set of partials we expect surface steps two atomic layers high (as indicated by the dashed line in the figure). Due to the interference of the indenter this is not possible. Instead we see that the atoms to the inside of the outer contact points have moved farther away from the indenter, creating an almost flat depression beneath the indenter, and the steps formed are not sharp. As a result there is a significant amount of distortion in the vicinity of the indenter which can be seen by viewing Fig. 12 at an oblique angle and looking along the $x$-direction beneath the indenter. Part of the

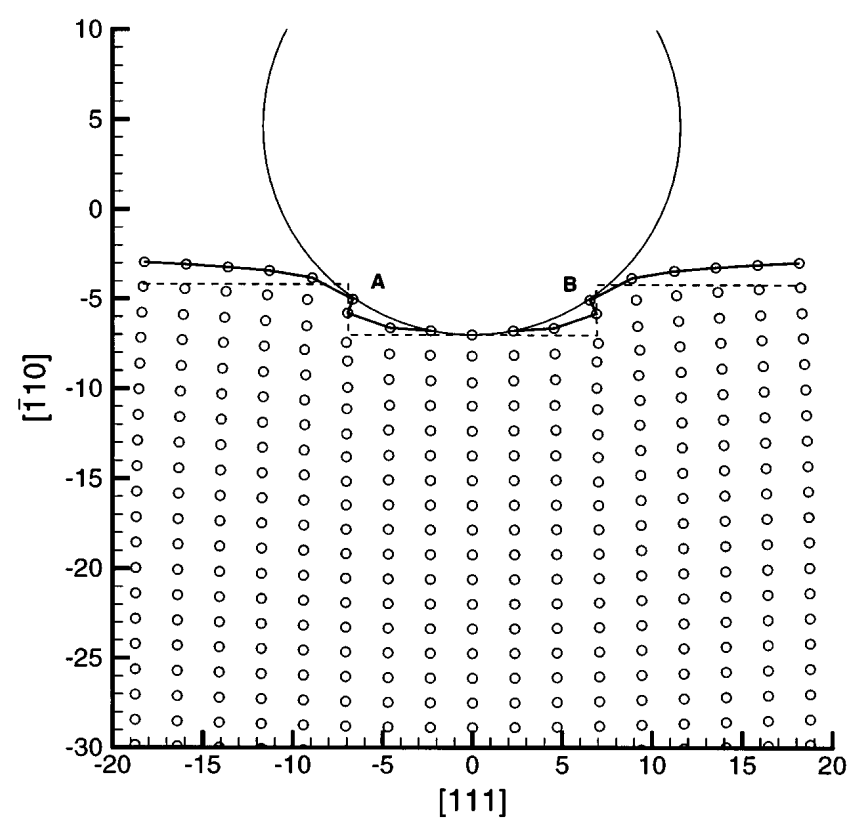

FIG. 12. Atomic structure beneath the cylindrical indenter after the emission of the second partial dislocation dipole which is out of the picture (dimensions in $\AA$ ). 
distortion is no doubt due to the perfect-stick boundary conditions imposed on the crystal. With a frictionless indenter the contact atoms ( $\mathrm{A}$ and $\mathrm{B}$ in the figure) could have moved out to the sides to better reproduce the stepped configuration with a resulting reduction in distortion.

\section{Elastic model for dislocation emission}

One can attempt to predict the width of contact and load for initial dislocation emission from elasticity theory. The shear stress beneath a cylindrical indenter is given in bipolar coordinates by, ${ }^{23}$

$$
\sigma_{x y}=-\frac{\mu y}{R(1-\nu)} \frac{r}{\sqrt{r_{1} r_{2}}} \sin \left[\theta-\frac{1}{2}\left(\theta_{1}+\theta_{2}\right)\right]
$$

where for a general point $(x, y), r=\sqrt{x^{2}+y^{2}}, r_{1}=$ $\sqrt{(x-a)^{2}+y^{2}}, r_{2}=\sqrt{(x+a)^{2}+y^{2}}, \theta=\tan ^{-1} y /$ $x, \theta_{1}=\tan ^{-1} y /(x-a)$, and $\theta_{2}=\tan ^{-1} y /(x+a)$ (see Fig. 6). The shear stress is not singular at the outer contact points (it is zero there) so we may consider the customary nucleation criterion of the form $\tau_{\max }=\tau_{c r}$ where $\tau_{\max }$ is the maximum shear stress and $\tau_{c r}$ is the critical shear stress threshold for dislocation nucleation. The maximum shear stresses do not lie along the planes beneath the contact points, as may have been expected, but rather they lie a little to the inside at $x= \pm \sqrt{3} a / 2$ and $y=-a / 2$. The maximum shear stress is,

$$
\tau_{\max }=\frac{\mu a}{4 R(1-\nu)}
$$

Applying the maximum stress criterion we find a contact half-width at emission (this would also be the emitted dipole half-width) equal to,

$$
a_{c r}=4(1-\nu) \frac{\tau_{c r}}{\mu} R
$$

and using the elastic relation between contact width and load (24) the critical load for emission follows as,

$$
P_{c r}=8 \pi(1-\nu) \mu R\left(\frac{\tau_{c r}}{\mu}\right)^{2}
$$

When the exact value of $\tau_{c r}$ is not known, an estimate for the critical values may be obtained by using $\tau_{c r} \approx \mu / 10$, in this case (29) and (30) reduce to $a_{c r}=0.4(1-\nu) R$ and $P_{c r}=1 / 4(1-\nu) \mu R$.

Relations (29) and (30) predict a linear scaling of both critical half-contact and critical load with indenter radius. It is of interest to validate this with the simulation. Figure 13 presents the critical load for a series of simulations, with indenter radius ranging from 4.48 to $69.84 \AA$. The simulation results appear as points and the solid line is a linear fit to them. We see that the scaling does indeed appear linear over the investigated range.

Ignoring, for the moment, the fact that the solid line does not intercept the $y$-axis at zero, we use the elastic prediction (30) to obtain the critical stress corresponding to its slope. The slope is $6.67 \mathrm{GPa}$ which corresponds to $\tau_{c r}=3.6 \mathrm{GPa}$. The elastic prediction using this value appears as the dashed line in the figure. The question remains, why the simulation results are offset by a constant value from the elastic prediction.

The reason for this discrepancy is the fact that the elasticity solution assumes a continuously increasing contact with applied loading, while the atomistic simulation is discrete. Thus relation (29) may predict any value while in actual fact atoms on the surface occupy discrete sites $n c$ where $n$ is an integer and $c$ is the $x$-spacing of atoms on the surface $(c=2.33 \AA$ for the current orientation). If we explicitly account for this by rounding $a_{c r}$ from (29) up to the nearest atomic site, the critical load follows from (24) as,

$$
P=\frac{\pi \mu n^{2} c^{2}}{2(1-\nu) R} \quad \text { for } R_{n-1}<R \leqslant R_{n}
$$

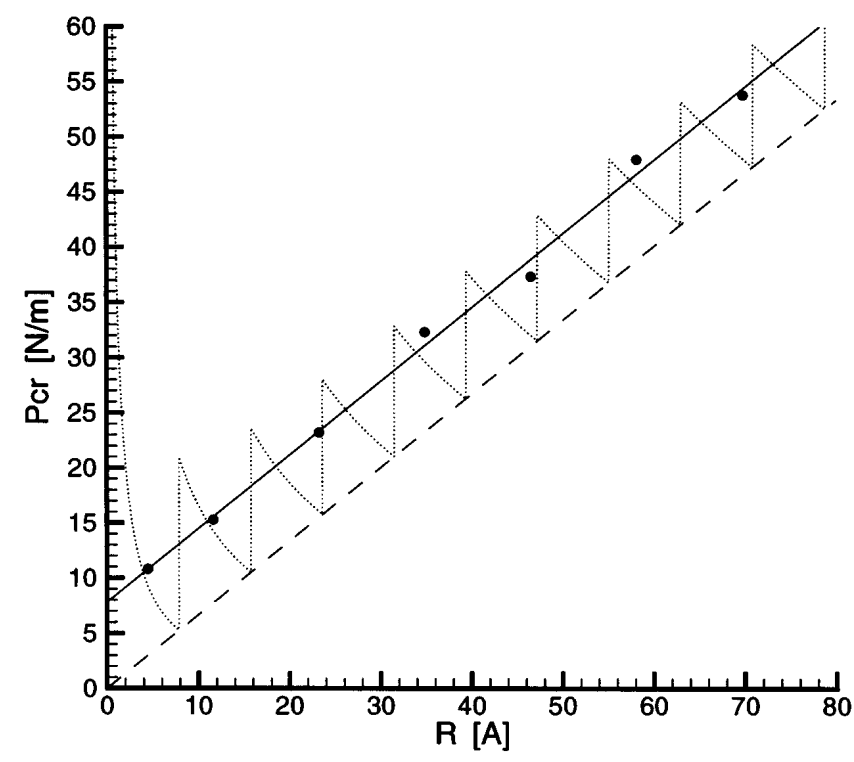

FIG. 13. Scaling of the critical emission load beneath a cylindrical indenter with indenter radius $R$ (the points correspond to simulation results, the solid line is a linear fit to these points, the dashed line is the elastic prediction, and the dotted line is the elastic result when accounting for lattice discreteness). 
where from (29), $R_{n}=\mu n c / 4(1-\nu) \tau_{c r}$. The amended relation in (31) appears as the dotted curve in Fig. 13. This curve shows the maximum scatter the lattice discreteness may be expected to generate, and we see that the simulation data lie well within this spread. It should be stressed that in the actual simulation the atoms on the surface move considerably due to elastic straining prior to being trapped by the indenter and thus the assumption that atoms are trapped at their reference sites $n c$ is not satisfied. The curve defined by (31) is thus just an indication of the type of effect lattice discreteness may play. It is also interesting to note that the curve diverges for $R<R_{1}$; this is the case where the critical half-contact predicted by elasticity theory is less than the atomic spacing on the surface.

At the heart of the preceding discussion was the assumption that a critical resolved shear stress is a valid criterion for dislocation emission, and indeed a value of $3.6 \mathrm{GPa}$ was obtained from the slope of our scaling curve. However, it must be clearly recognized that this is only an effective value as it applies to the small strain linear elastic solution. In the simulations, the actual resolved shear stresses at emission did not equal 3.6 GPa and were not even constant. In fact, the observed values appeared to decrease with indenter radius ranging from 4.2 $\mathrm{GPa}$ for the smallest indenter to $3.2 \mathrm{GPa}$ for the largest. The resolved shear strains did not show this trend, and it is currently being investigated whether an emission criterion based on a critical resolved shear strain would be more useful. Such a criterion may be more physically meaningful because the resolved shear strain is a measure of the incipient slip prior to dislocation emission. A critical shear strain may characterize the loss of stability of the incipient slip profile at the instant of emission. Note that for the elastic solution the shear stresses and strains are proportional and thus the critical values (29) and (30) would be exactly the same for a critical strain criterion with $\tau_{c r} / \mu$ replaced by $2 \epsilon_{c r}$. This may explain the qualitative success of the elastic model in predicting the correct scaling.

\section{Unloading}

Next, we turn to the unloading phase. Unloading was effected by reversing the direction of motion of the atoms beneath the indenter. These atoms are now slowly pulled up and released when found to be held by a tensile load to the indenter face. The unloading curve can be seen in Fig. 8 from points $F$ to $J$. A linear unloading curve would be expected for the case where a constant contact area is maintained during unloading, ${ }^{33}$ as indicated by the dashed line. Instead we see that the unloading curve that is obtained is composed of short, nearly linear segments with changes of slope at the points where atoms are released from the indenter. At the start of unloading seven atoms (per unit repeat distance in the out-of-plane direction) are in contact with the indenter. A series of snapshots of the atomic structure beneath the indenter during unloading is given in Fig. 14. At $G$ $(6.6 \AA)$ the outer atoms are released, at $H(5.6 \AA)$ the pair of atoms to the left and right of the central atom are released, at $I(4.2 \AA)$ the central atom is released, and finally at $J$ the remaining pair is released. At the end, a rectangular dimple $14 \AA$ wide and $2.8 \AA$ deep (and infinite in the out-of-plane direction) is left behind as a permanent deformation. The residual depth is essentially equal to the magnitude of the Burgers vector, $2.85 \AA$. The small difference is due to surface relaxation.

At the end of the unloading process, the edge dislocation dipole remains in the crystal bulk, trapped by lattice friction. Without the assistance of thermal vibration, absent in this zero temperature simulation, the image forces alone are not sufficiently large to overcome the Peierls barrier. It thus appears that in this case the Peierls barrier alone is responsible for the irreversibility of the deformation. The rectangular indenter was not
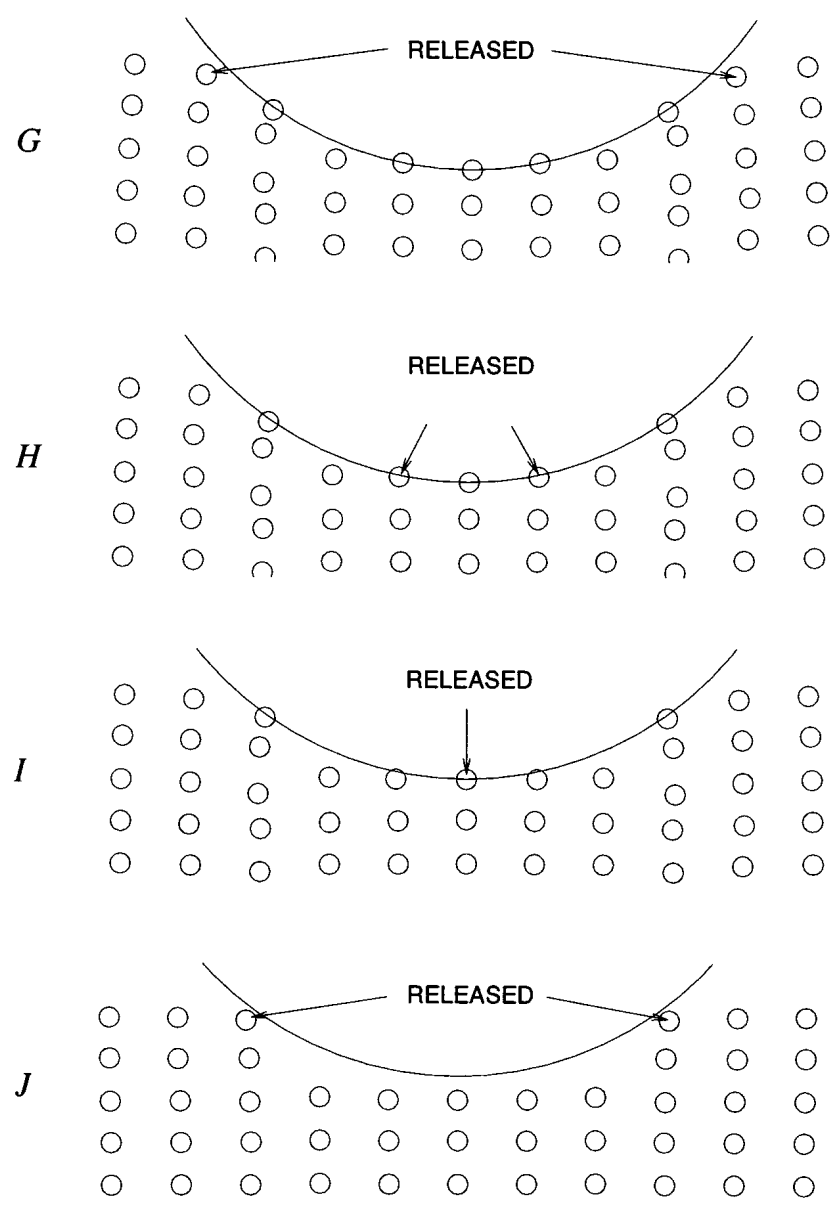

FIG. 14. Atomic structure beneath the cylindrical indenter during the unloading steps $G$ through $J$, as indicated in the load displacement figure. 
unloaded, so it is not known whether the same effect would be observed in that case.

\section{NANOINDENTATION IN THE TWINNING ORIENTATION}

In the second orientation that was investigated we indented into a (111) face of the crystal with the global $x$-direction coinciding with the [1 $\overline{1} 2]$ crystallographic direction. The most favorable available slip plane in the analysis is now a $(\overline{1} \overline{1} 1)$ plane oriented at $19.47^{\circ}$ with respect to the indentation direction with the slip direction along this plane corresponding to the [112] direction. The crystal orientation and geometry were presented earlier in the schematic in Fig. 1(b).

\section{A. Load-displacement response and partial dislocation nucleation}

The resulting load-displacement curve is presented in Fig. 15. This result is contrasted with the curve obtained for the rectangular indentation in the dislocation orientation which appears as a dashed line in the figure. The two curves are clearly quite different with the new curve lacking the large load drops associated with dislocation emission seen in the previous case. Instead, in this orientation the curve is linear throughout with occasional small steps at nearly constant load associated with partial dislocation emission, as will be explained presently.

Whereas in the previous configurations studied, dislocation emission was always a discrete event accompanied by a marked load drop, in this orientation the

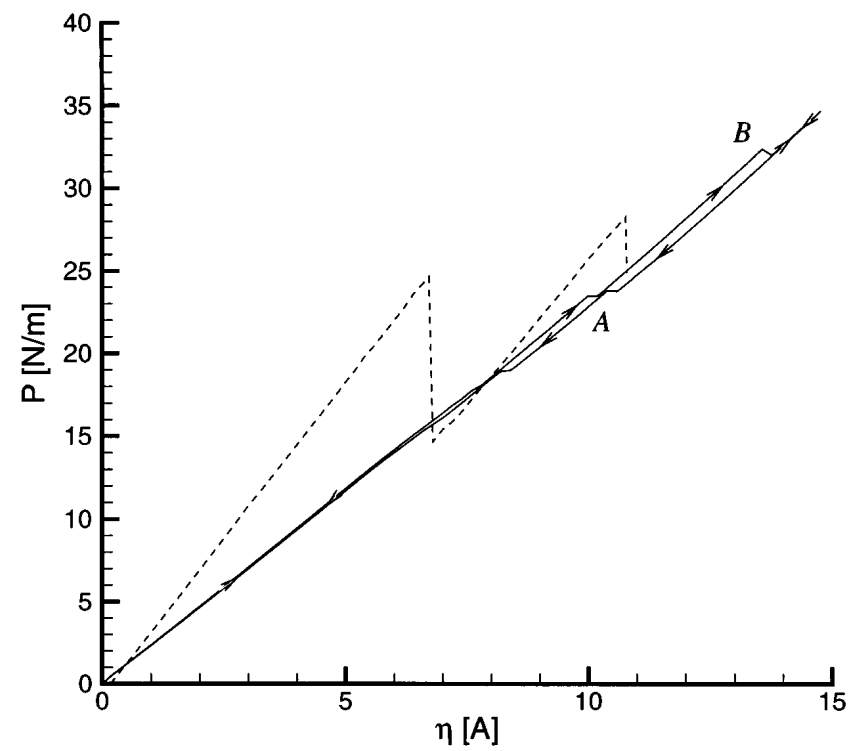

FIG. 15. Computed load-displacement curve for nanoindentation into a (111) face of an aluminum thin film (solid line), superimposed on the earlier curve computed for the dislocation orientation. first dislocation is nucleated gradually during the linear portion of the load-displacement curve up to $A$. There is no definite emission event and no discernible load drop associated with this gradual nucleation. Instead, slip continuously builds across the ( $\overline{1} \overline{1} 1)$ plane to the left of the indenter tip, gradually forming a partial dislocation. No out-of-plane displacements were generated since the partial is of a pure edge type, referred to as a $90^{\circ}$ Shockley partial. This dislocation has a $1 / 6$ [112] Burgers vector and a $[110]$ line direction perpendicular to the $x y$ plane of the analysis. A view of the atomic structure beneath the indenter after this partial is fully formed and just before the emission of a second partial at step $A$ is given in Fig. 16. The partial dislocation at the tip of the slipped region is indicated along with its highlighted extra halfplane and some relevant crystallographic directions. The slip plane appears as a dashed line.

The nucleated partial is of type $D \gamma$, as denoted in the (1) 11$)$ face of the Thompson tetrahedron ${ }^{22}$ presented in Fig. 17. In the figure, $A B$ corresponds to the line direction (the out-of-plane direction in the analysis). If the deformation were to follow along the same lines as the "dislocation orientation" presented in Sec. IV. A, we would expect a second partial dislocation to be nucleated to complete the dissociated structure. In this case, the full dislocation would be a $60^{\circ}$ dislocation (i.e., the Burgers vector and dislocation line would be at a $60^{\circ}$ angle) with a [110] line direction. From the Thompson tetrahedron we see that this dislocation can have two possible Burgers vectors: either [101] ( $D A$ in the figure) or [011] ( $D B$ in the figure). The two options are symmetric and equally probable. For the [101] dislocation the second partial would be a $30^{\circ}$

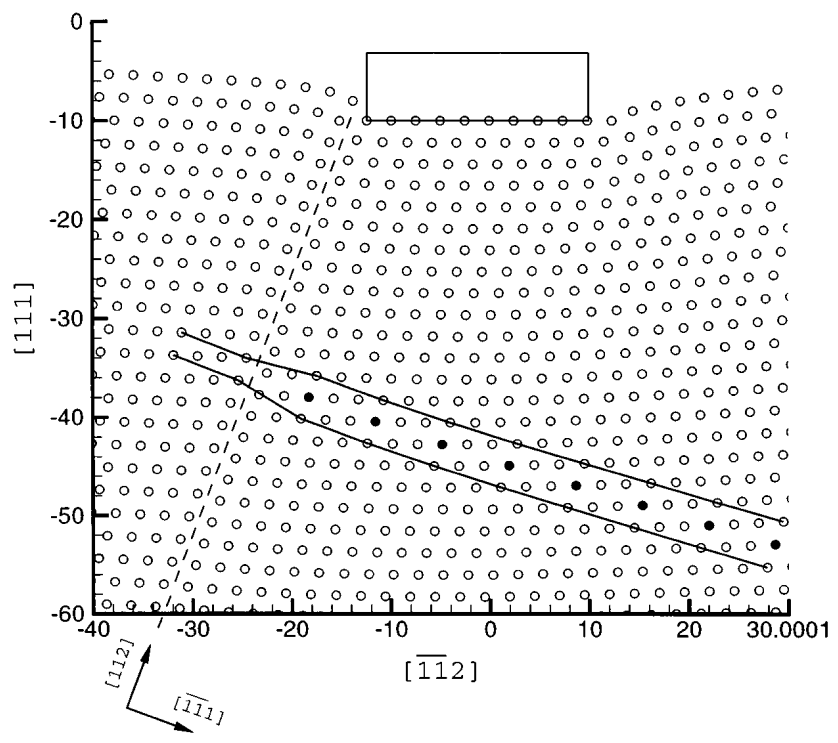

FIG. 16. Atomic structure beneath the indenter just before step $A$ (dimensions are in $\AA$ ). 


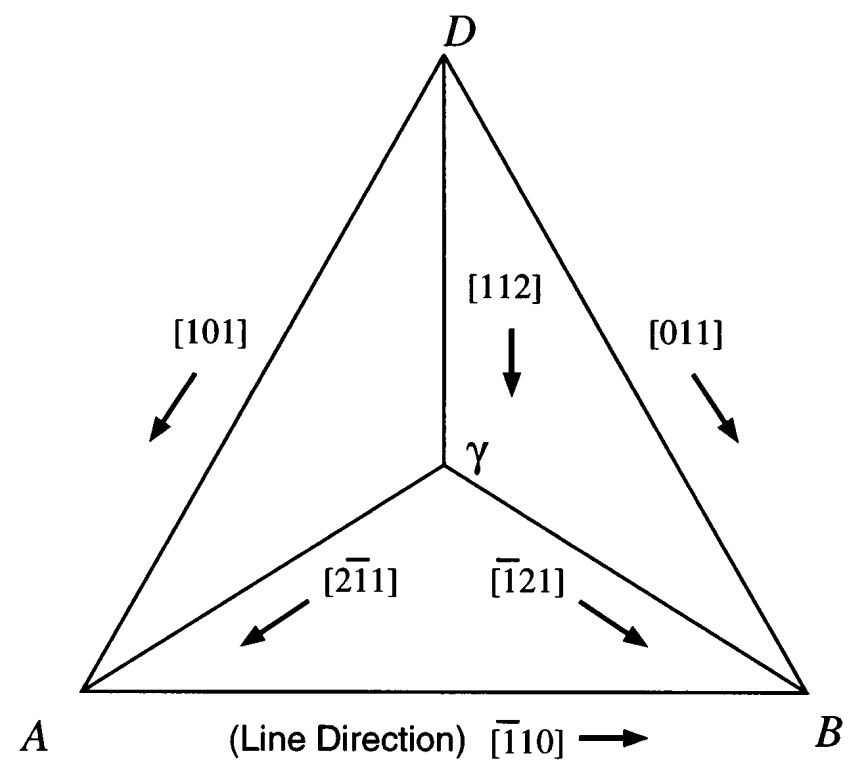

FIG. 17. The $(\overline{1} \overline{1} 1)$ plane of the Thompson tetrahedron.

Shockley with Burgers vector [2 $\overline{1} 1]$ ( $\gamma A$ in the figure), while for the [011] dislocation the Burgers vector would be $[121](\gamma B$ in the figure). These partials are of mixed screw-edge type and if formed in the $x y$-plane of analysis (which is perpendicular to the Thompson plane in the figure) would carry out-of-plane components in opposite directions.

As the deformation progresses the scenario envisioned above does not transpire. Instead of nucleating the second partial on the same plane to form a perfect dissociated dislocation, additional dislocations are nucleated on adjacent planes of the same type as the original $D \gamma$ partial. The first of these additional nucleations occurs at the end of step $A$ in the load-displacement figure (Fig. 15) at an accumulated indentation depth of $10.2 \AA$ and the second occurs at step $B$ at an indentation depth of $13.8 \AA$. A series of snapshots of the successive nucleations are shown in isometric view in Fig. 18. To create the figure all atoms are projected onto the $x y$ plane and the plane is then rotated in 3D space to afford the reader a clearer view of the structure beneath the indenter. Frame (a) contains a snapshot of the structure at the beginning of step $A$ (indentation $=10.0 \AA$ ), showing the partial dislocation with a stacking fault in its wake.
Frame (b) taken at the end of the step indicates a second nucleation has occurred on a plane adjacent and above the previous one. It forms at about the same distance from the surface where the previously nucleated partial originally formed and makes a step in the structure we begin to see emerging. Thus the "kinked" region goes from a thickness of two atomic spacings near the surface to only one near the bottom where the original partial is still located. The structure near the surface corresponds to a microtwin with a ( $\overline{1} \overline{1} 1)$ twinning plane and a [112] twinning direction. In frame (c) a third partial has been emitted, increasing the thickness of the twinned region. The typical "needle-like" structure associated with deformation twinning ${ }^{34,35}$ is beginning to emerge with the discrete steps at which the needle becomes thinner, corresponding to the positions of the emitted partials. The leading partial situated at the tip of the needle is now out of the picture. Finally, Fig. 19 shows the atomic structure beneath the indenter at the end of the simulation at an accumulated indentation depth of $14.8 \AA$ A. The twinning planes have been indicated by lines and the needle-like morphology is clearly discernible.

\section{B. Deformation twinning}

The appearance of deformation twinning is a surprising result since aluminum is normally regarded as a metal that does not exhibit deformation twinning. ${ }^{34}$ Recently, however, deformation twinning has been observed experimentally in aluminum at the tips of cracks in thin foils. ${ }^{36}$ The fact that in both the experiment and our simulation a two-dimensional state of deformation exists (plane stress for the crack and plane strain for the indenter) suggests that perhaps the appearance of deformation twinning in these cases is tied to the 2D kinematic constraints. It has also been observed that other fcc metals that do not normally deformation twin at certain temperatures, like gold and silver, do so under the constraining action of a nanoindenter. ${ }^{25}$ Deformation twinning may have been further facilitated in the current simulation since it was carried out at zero temperature and it is well known that deformation twinning becomes more favorable with decreasing temperature. Finally, it is also possible that the twinning observed here is an artifact of the simulation resulting from the limitations

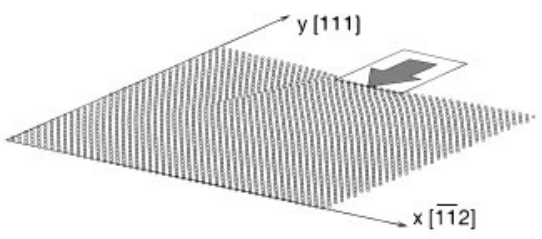

(a)

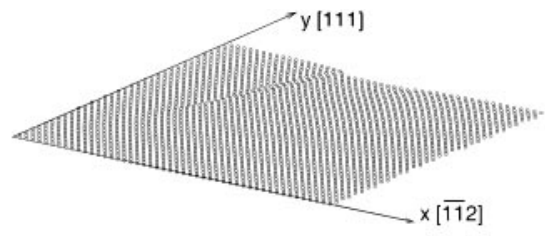

(b)

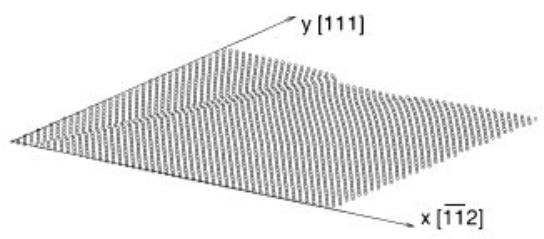

(c)

FIG. 18. Isometric views of the structure beneath the indenter at (a) just prior to step $A$, (b) just after step $A$, and (c) just after step $B$. 


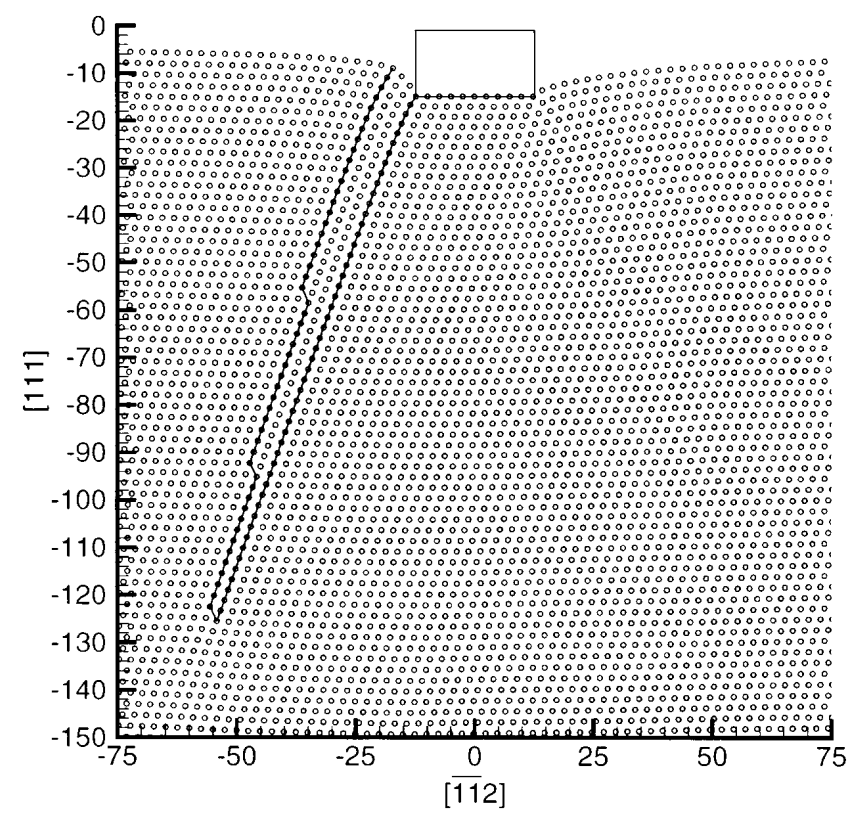

FIG. 19. Final atomic structure beneath the indenter in the twinning orientation, showing a deformation twin (dimensions in $\AA$ ).

of the embedded atom potential used to characterize the material.

The observation of deformation twinning for this orientation is especially interesting in light of the recent debate in the literature concerning the plastic mechanisms at work during very small-scale indentations into (111) surfaces of fcc crystals. Some recent experimental work by Pharr and Oliver ${ }^{9}$ appears to indicate the lack of near-surface dislocation activity during small-scale indentation in silver. It was found that the dislocation rosette patterns normally observed on the surface after indentation disappear for very small indentations. The authors were unable to experimentally determine the active plastic mechanism, but they rule out diffusion and postulate that either the dislocations are quickly annealed away, that they move down into the bulk without emerging at the surface, or that there is some unknown mechanism at work. Belak et al. ${ }^{12}$ in their MD simulations of nanoindentation in copper support the view that no dislocation activity takes place at small-scale indentation for the (111) orientation. In their case plastic deformation was accommodated by the movement of individual atoms to the surface or to interstitial positions with no dislocation activity apparent. By contrast, in recent MD simulations by Kelchner et $a l .{ }^{37}$ of nanoindentation in gold, dislocation activity was observed in the (111) orientation. The resulting structure after indentation contained partial dislocations, stacking faults, and stair rod dislocations joining nonparallel $\{111\}$ planes. Finally, in the present simulation, as discussed above, deformation twinning was observed.
These discrepancies in the simulation results may be due to real physical differences in the systems investigated, or they may simply indicate limitations of the various models used in the analyses. It appears that more work needs to be done to clarify this issue.

\section{Unloading}

Finally, a word about unloading. The unloading portion of the load-displacement curve (see Fig. 15) follows the loading curve back down closely with the exception that the steps, which now correspond to the annihilation of the partial dislocations which travel back up to the surface, appear in different places. At the end, upon full removal of the load, the crystal is restored to its perfect structure with no residual deformation. This is not surprising considering the presence of the stacking faults which tend to pull the dislocations back to minimize their energy. This has been observed experimentally in other materials such as calcite where small twins nucleated by indentation disappear upon removal of the load. ${ }^{38}$

\section{CONCLUSIONS}

This paper has presented a detailed computational investigation of the early stages of plastic formation observed under the action of a nanoindenter in an aluminum thin film. The quasicontinuum method ${ }^{14,17}$ was used to model the response, allowing for the study of a much larger system than standard atomistic techniques would enable while retaining atomic resolution where necessary. An embedded atom potential due to Ercolessi and Adams ${ }^{21}$ was used to characterize the aluminum.

Three different configurations were studied involving different crystallographic orientations and indenter geometries. Indentation into a (110) surface resulted in the emission of dissociated edge dislocation dipoles from beneath the indenter. The emission events were accompanied by sudden drops in the indenter load. Upon unloading, the dislocation dipoles remain trapped in the crystal as a result of lattice friction. In contrast, indentation into a (111) surface was accommodated by a deformation twinning mechanism. In this case, the load-displacement curve is nearly linear with small steps at nearly constant load as successive Shockley partial dislocations are emitted in the twinning process. When the crystal is unloaded, the partial dislocations are pulled back to the surface, undoing the twinning operation, and restoring the crystal to its perfect structure. The appearance of deformation twinning is a surprising result since $\mathrm{Al}$ is not a metal commonly associated with this mode of deformation. The result may be a consequence of kinematic constraints imposed by the $2 \mathrm{D}$ nature of the simulation. 
The Peierls stress for an edge dislocation in aluminum was estimated from the equilibrium depth at which the emitted dislocation dipole settled. It was found to be $2.5 \times 10^{-4} \mu$ (where $\mu$ is the shear modulus) for the Ercolessi-Adams EAM potential used. In addition, the density of geometrically necessary dislocations beneath the rectangular indenter was computed and compared to the simple model of François et al. ${ }^{31}$ It was found that elastic effects and the presence of the dislocations emitted earlier, both neglected by the François model, are significant. The computed density was $30 \%$ smaller than the model prediction.

One of the provocative questions raised by this study relates to the significance of the critical resolved shear stress as a criterion for dislocation emission. When studying the effect of indenter radius on dislocation emission, a linear scaling was found between critical load and indenter radius, as predicted by elasticity theory where a critical stress criterion was assumed. However, in the simulations, the critical resolved shear stress at emission was not a constant. Instead, it decreased with indenter radius from a value of 4.2 to $3.2 \mathrm{GPa}$. This is not thought to be a simulation size effect because the ranges of radii investigated are all very small with respect to the system size and the emission is highly localized to the indenter tip. There are indications that it may be the critical resolved shear strain that is more fundamental, and this is currently being investigated.

The above question is particularly important since in addition to rationalizing experimental results, an important goal of atomic-scale simulations of nanoindentation is to obtain physically based criteria for dislocation nucleation beneath indenters. The objective here is to make contact with larger-scale dislocation dynamics models. ${ }^{39}$ Recently, such a model coupled with a finite element simulation has been effectively used to study the dislocation structure induced by nanoindentation. ${ }^{40} \mathrm{How}-$ ever, since the discrete elements of these simulations are dislocation segments, dislocation nucleation must be introduced by a phenomenological rule. As stated above, it is one of the goals of atomic-scale simulations to obtain such rules, and this is currently being pursued.

The investigation presented in this paper is of a theoretical and computational nature. While there are many recent nanoindentation experiments in a variety of materials, to our knowledge there have been no nanoindentation experiments done using the two-dimensional knife-edge indenter envisaged here and hence it was not possible to make any direct comparisons with experimental data. While, clearly, such an experiment would be technically far more challenging than simpler 3D indentation due to tip fabrication, surface roughness, and tip/substrate alignment issues, it could offer significant advantages in the interpretation of results due to the simpler geometry and simpler resulting dislocation structure.

\section{ACKNOWLEDGMENTS}

We are grateful to Bernard Budianski, Gilles Canova, Marc Fivel, Bill Gerberich, Ladislas Kubin, Erica Lilleodden, David Rodney, and Vijay Shenoy for many stimulating discussions. In addition, we gratefully acknowledge support by the AFOSR through Grant F49620-95-I-0264, the NSF through Grants CMS9414648 and DMR-9632524, and the MRSEC program at Brown University.

\section{REFERENCES}

1. W. D. Nix, Metall. Trans. A 20A, 2217 (1989).

2. A.P. Sutton and J. B. Pethica, J. Phys.: Condens. Matter 2, 5317 (1990).

3. F. A. McClintock and A. S. Argon, Mechanical Behavior of Materials (Addison-Wesley, Reading, MA, 1966), Chap. 13.

4. S. J. Sharp, M. F. Ashby, and N. A. Fleck, Acta Metall. Mater. 41, 685 (1993).

5. W. W. Gerberich, J. C. Nelson, E. T. Lilleodden, P. Anderson, and J. T. Wyrobek, Acta Mater. 44, 3585 (1996).

6. N. Gane and F. P. Bowden, J. Appl. Phys. 39, 1432 (1968).

7. R. Nowak, C. L. Li, and S. Maruno, J. Mater. Res. 12, 64 (1997).

8. P. Tangyunyong, R. C. Thomas, J. E. Houston, T. A. Michalske, R. M. Crooks, and A. J. Howard, Phys. Rev. Lett. 71, 3319 (1993).

9. G. M. Pharr and W. C. Oliver, J. Mater. Res. 4, 94 (1989).

10. U. Landman, W. D. Luedtke, N. A. Burnham, and R. J. Colton, Science 248, 454 (1990).

11. J. S. Kallman, W. G. Hoover, C. G. Hoover, A. J. De Groot, S. M. Lee, and F. Wooten, Phys. Rev. B 47, 7705 (1993).

12. J. Belak, J.N. Glosli, D. B. Boercker, and I.F. Stowers, in Modelling and Simulation of Thin-Film Processing, edited by D. Srolovitz, C.A. Volkert, M. J. Fluss, and R. J. Kee (Mater. Res. Soc. Symp. Proc. 389, Pittsburgh, PA, 1995), p. 181.

13. E. B. Tadmor, The Quasicontinuum Method, Ph.D. Thesis, Brown University, 1996.

14. E. B. Tadmor, M. Ortiz, and R. Phillips, Philos. Mag. A73, 1529 (1996).

15. E. B. Tadmor, R. Phillips, and M. Ortiz, Langmuir 12, 4529 (1996).

16. V.B. Shenoy, R. Miller, E. B. Tadmor, R. Phillips, and M. Ortiz, Phys. Rev. Lett. 80, 742 (1998).

17. V.B. Shenoy, R. Miller, E. B. Tadmor, D. Rodney, R. Phillips, and M. Ortiz, J. Mech. Phys. Solids 47, 611 (1999).

18. O.C. Zienkiewicz and R. L. Taylor, The Finite Element Method, 4th ed. (McGraw-Hill, London, 1989).

19. M. S. Daw and M. I. Baskes, Phys. Rev. Lett. 50, 1285 (1983).

20. M. S. Daw, Many-Atom Interactions in Solids, Springer Proceedings in Physics (Springer-Verlag, Berlin, 1990), Vol. 48, p. 48.

21. F. Ercolessi and J. Adams, Europhys. Lett. 26, 583 (1994).

22. J.P. Hirth and J. Lothe, Theory of Dislocations, 2nd ed. (Krieger, Malabar, FL, 1992), pp. 317, 424.

23. N. I. Muskhelishvili, Some Basic Problems of the Mathematical Theory of Elasticity, 3rd ed. (P. Noordhoff Ltd., Groningen, The Netherlands, 1953), pp. 481-483.

24. A. V. Kulkarni and B. Bhushan, Mater. Lett. 29, 221 (1996).

25. Q. Ma and D. R. Clarke, J. Mater. Res. 10, 853 (1995).

26. M. J. Mills and P. Stadelmann, Philos. Mag. A 60, 355 (1989).

27. J. Friedel, Dislocations (Addison-Wesley, Reading, MA, 1967), pp. 40, 45, 54, 230.

28. T. Kosugi and T. Kino, Mater. Sci. Eng. A 164, 368 (1993).

29. T. Egami and D. Srolovitz, J. Phys. F: Met. Phys. 12, 2141 (1982).

30. V. Vitek and T. Egami, Phys. Status Solidi B 144, 145 (1987). 
31. D. François, A. Pineau, and A. Zaoui, Comportement Mecanique des Materiaux (elasticite et plasticite), 3rd ed. (Hermes, Paris, 1995), pp. 189-190.

32. W. D. Nix, Mater. Sci. Eng. A234, 37 (1997).

33. M. F. Doerner and W.D. Nix, J. Mater. Res. 1, 601 (1986).

34. J. A. Venables, in Deformation Twinning, Proceedings of the Metallurgical Society Conference, edited by R. E. Reed-Hill (Gordon and Breach Science Publishers, 1963), Vol. 25, p. 77.

35. P. Rosakis and H. Tsai, Mech. Mater. 17, 245 (1994).

36. R. C. Pond and L. M.F. Garcia-Garcia, Inst. Phys. Conf. Ser., No. 61, 495 (1981).
37. C. L. Kelchner, S. J. Plimpton, and J. C. Hamilton, Phys. Rev. B 58, 11085 (1998).

38. A. Kelly and G. W. Groves, Crystallography and Crystal Defects (Addison-Wesley, Reading, MA, 1970), p. 311.

39. L. Kubin, G. Canova, M. Condat, B. Devincre, V. Pontikis, and Y. Bréchet, Solid State Phenom. 23 \& 24, 455 (1992).

40. M. Fivel, M. Verdier, and G. Canova, Mater. Sci. Eng. A234-236, 923 (1997). 